\title{
One-Pot Asymmetric Synthesis of Acyclic Chiral Epoxy Alcohols via Tandem Vinylation-Epoxidation with Dioxygen
}

\author{
Alice E. Lurain, Patrick J. Carroll and Patrick J. Walsh* \\ Contribution from the P. Roy and Diana T. Vagelos Laboratories, Department of Chemistry, \\ University of Pennsylvania, 231 South $34^{\text {th }}$ Street, Philadelphia, PA 19104-6323 \\ Email: pwalsh@sas.upenn.edu
}

\section{Supporting Information}

General Methods

Procedures and Characterization of 1-28

References

${ }^{1} \mathrm{H}$ and ${ }^{13} \mathrm{C}\left\{{ }^{1} \mathrm{H}\right\}$ NMR Spectra of 1-28 
General Methods. All reactions were carried out under a nitrogen atmosphere with oven-dried glassware. The progress of all reactions was monitored by thin-layer chromatography to ensure the reactions had reached completion. All manipulations involving dicyclohexylborane and dialkylzinc reagents were carried out using an inert atmosphere in a drybox or by using standard Schlenk or vacuum line techniques. Dichloromethane and hexanes were dried through alumina columns. All aldehydes were distilled or recrystallized and sublimed prior to use and stored under $\mathrm{N}_{2}$. 230-400 mesh silica gel was used for air-flashed chromatography. The ${ }^{1} \mathrm{H}$ NMR and ${ }^{13} \mathrm{C}\left\{{ }^{1} \mathrm{H}\right\} \mathrm{NMR}$ spectra were obtained on an AM-500 Fourier transform NMR spectrometer at 500 and $125 \mathrm{MHz}$, respectively. Chemical shifts are reported in units of parts per million downfield from tetramethylsilane, and all coupling constants are reported in Hertz. Analysis of enantiomeric excess was performed using chiral HPLC with a Chiralcel OD$\mathrm{H}$ column or by chiral capillary gas chromatography with a Beta-DEX column. Absolute configuration was determined by comparison of optical rotation to literature data for known compounds.

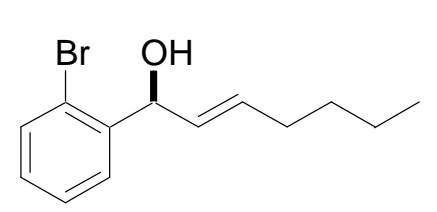

(S)-1-(2-Bromo-phenyl)-hept-2-en-1-ol (4). General Procedure A. A $10 \mathrm{~mL}$ Schlenk flask was charged with $\mathrm{Cy}_{2} \mathrm{BH}$ (98 mg, $0.55 \mathrm{mmol}$ ), prepared according to Oppolzer's procedure, ${ }^{1}$ and $1.5 \mathrm{~mL}$ of hexanes. 1-Hexyne $(64 \mu \mathrm{L}, 0.55$ mmol) was added dropwise, and the homogenous reaction mixture was stirred for $30 \mathrm{~min}$ at room temp. After cooling the reaction flask to $-10{ }^{\circ} \mathrm{C}$, (-)-MIB (4.8 mg, $0.02 \mathrm{mmol})$ was added, followed by $\mathrm{Et}_{2} \mathrm{Zn}(0.55 \mathrm{~mL}, 2.0 \mathrm{M})$, then $o$ bromobenzaldehyde $(58 \mu \mathrm{L}, 0.50 \mathrm{mmol})$ dropwise. The reaction was stirred at $-10{ }^{\circ} \mathrm{C}$ for $2 \mathrm{~h}$ and quenched with $2 \mathrm{~mL} \mathrm{H}_{2} \mathrm{O}$. The organic and aqueous layers were separated, and the aqueous layer was extracted with hexanes $(3 \mathrm{X} 5 \mathrm{~mL})$. The combined organic layers were then washed with $5 \mathrm{~mL} \mathrm{H}_{2} \mathrm{O}$ and dried over $\mathrm{MgSO}_{4}$. The filtrate was concentrated in vacuo, and the residue was chromatographed on silica (5\% ethyl acetate in hexanes) to afford the title compound in $77 \%$ yield (104 $\mathrm{mg}, 0.39 \mathrm{mmol})$ as a colorless oil. $[\alpha]_{\mathrm{D}}{ }^{20}=-31.3\left(c=3.2, \mathrm{CHCl}_{3}, 93 \%\right.$ ee $) ;{ }^{1} \mathrm{H} \mathrm{NMR}\left(\mathrm{CDCl}_{3}, 500 \mathrm{MHz}\right): \delta 0.86(\mathrm{t}, 3 \mathrm{H}$, $J=7.1 \mathrm{~Hz}), 1.25-1.37(\mathrm{~m}, 4 \mathrm{H}), 2.03(\mathrm{dt}, 2 \mathrm{H}, J=7.0,6.7 \mathrm{~Hz}), 2.11(\mathrm{br} \mathrm{s}, 1 \mathrm{H}), 5.51(\mathrm{~d}, 1$ $\mathrm{H}, J=6.1 \mathrm{~Hz}), 5.58(\mathrm{dd}, 1 \mathrm{H}, J=15.2,6.4 \mathrm{~Hz}), 5.78(\mathrm{dt}, 1 \mathrm{H}, J=15.2,6.7 \mathrm{~Hz}), 7.10(\mathrm{t}, 1 \mathrm{H}$ $J=7.6 \mathrm{~Hz}), 7.31(\mathrm{t}, 1 \mathrm{H}, J=7.4 \mathrm{~Hz}), 7.50(\mathrm{~d}, 1 \mathrm{H}, J=7.9 \mathrm{~Hz})$, and $7.53(\mathrm{~d}, 1 \mathrm{H}, J=7.7$ $\mathrm{Hz})$ ppm; ${ }^{13} \mathrm{C}\left\{{ }^{1} \mathrm{H}\right\}$ NMR $\left(\mathrm{CDCl}_{3}, 125 \mathrm{MHz}\right): \delta 13.9,22.2,31.3,31.9,73.5,122.4$, 127.70, 127.74, 128.8, 130.2, 132.7, 133.5, and 142.2 ppm; IR (neat): 3360, 3060, 2956,

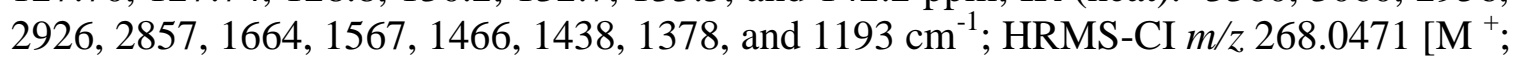
calcd for $\mathrm{C}_{13} \mathrm{H}_{17} \mathrm{BrO}$ : 268.0463].

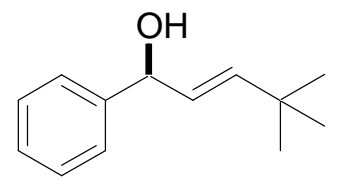

(S)-4,4-Dimethyl-1-phenyl-pent-2-en-1-ol (1). The product was prepared by General Procedure A using $1.07 \mathrm{~g}(6.0 \mathrm{mmol}) \mathrm{Cy}_{2} \mathrm{BH}$, $739 \mu \mathrm{L}$ (6.0 mmol) 2,2-dimethyl-1-butyne, $3.0 \mathrm{~mL}$ (6.0 mmol, 2.0 $\mathrm{M}$ in toluene) $\mathrm{Me}_{2} \mathrm{Zn}, 24.0 \mathrm{mg}(0.1 \mathrm{mmol})(-)-\mathrm{MIB}$, and $510 \mu \mathrm{L}$ (5.0 mmol) benzaldehyde. The crude product was purified by column chromatography on silica (20\% ethyl acetate in hexanes) to afford the title compound as a colorless oil in $86 \%$ yield $(819 \mathrm{mg}, 4.3 \mathrm{mmol}) .[\alpha]_{\mathrm{D}}{ }^{20}=+40.6\left(c=1.00, \mathrm{CHCl}_{3}, 96 \%\right.$ ee $) ;{ }^{1} \mathrm{H} \mathrm{NMR}$ $\left(\mathrm{CDCl}_{3}, 500 \mathrm{MHz}\right): \delta 1.14(\mathrm{~s}, 9 \mathrm{H}), 2.54(\mathrm{~s}, 1 \mathrm{H}), 5.19(\mathrm{~d}, 1 \mathrm{H}, J=6.8 \mathrm{~Hz}), 5.65(\mathrm{dd}, 1 \mathrm{H}, J$ 
$=15.4,6.9 \mathrm{~Hz}), 5.86(\mathrm{~d}, 1 \mathrm{H}, J=15.9 \mathrm{~Hz})$, and 7.34-7.45 (m, 5H) ppm; ${ }^{13} \mathrm{C}\left\{{ }^{1} \mathrm{H}\right\} \mathrm{NMR}$ $\left(\mathrm{CDCl}_{3}, 125 \mathrm{MHz}\right): \delta 30.0,33.3,75.7,126.7,127.8,128.7,128.9,143.7$, and $144.1 \mathrm{ppm}$; IR (neat): 3346, 3086, 3063, 3029, 2959, 2902, 2866, 1494, 1476, and $1453 \mathrm{~cm}^{-1}$; HRMS-CI $m / z, 190.1356\left[\mathrm{M}^{+}\right.$; calcd for $\left.\mathrm{C}_{13} \mathrm{H}_{18} \mathrm{O}: 190.1358\right]$.

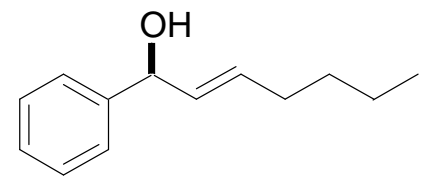

(S)-1-Phenyl-hept-2-en-1-ol (2). The product was prepared by General Procedure A using $2.14 \mathrm{~g}(12.0 \mathrm{mmol}) \mathrm{Cy}_{2} \mathrm{BH}, 1.4$ $\mathrm{mL}(12.0 \mathrm{mmol})$ 1-hexyne, $6 \mathrm{~mL}(12.0 \mathrm{mmol}, 2.0 \mathrm{M}$ in toluene) $\mathrm{Me}_{2} \mathrm{Zn}, 48 \mathrm{mg}(0.2 \mathrm{mmol})(-)-\mathrm{MIB}$, and $1.01 \mathrm{~mL}$ (10.0 mmol) benzaldehyde. The crude product was purified by column chromatography on silica (5\% ethyl acetate in hexanes) to afford the title compound as a colorless oil in $85 \%$ yield $(1.62 \mathrm{~g}, 8.52 \mathrm{mmol}) .[\alpha]_{\mathrm{D}}{ }^{20}=+38.3\left(c=1.01, \mathrm{CHCl}_{3}, 95 \%\right.$ ee $) ;{ }^{1} \mathrm{H} \mathrm{NMR}$ $\left(\mathrm{CDCl}_{3}, 500 \mathrm{MHz}\right): \delta 0.85(\mathrm{t}, 3 \mathrm{H}, J=7.1 \mathrm{~Hz}), 1.22-1.37(\mathrm{~m}, 4 \mathrm{H}), 1.81(\mathrm{br} \mathrm{s}, 1 \mathrm{H}), 2.02$ $(\mathrm{dd}, 2 \mathrm{H}, J=7.0,6.5 \mathrm{~Hz}), 5.13(\mathrm{~d}, 1 \mathrm{H}, J=6.6 \mathrm{~Hz}), 5.63(\mathrm{dd}, 1 \mathrm{H}, J=15.3,6.7 \mathrm{~Hz}), 5.73$ (dt, $1 \mathrm{H}, J=15.3,6.6 \mathrm{~Hz})$, and 7.22-7.34 (m, 5H) ppm; ${ }^{13} \mathrm{C}\left\{{ }^{1} \mathrm{H}\right\}$ NMR $\left(\mathrm{CDCl}_{3}, 125\right.$ $\mathrm{MHz}): \delta 13.9,22.2,31.2,31.8,75.1,126.1,127.3,128.4,132.2,132.7$, and $143.4 \mathrm{ppm}$; IR (neat): $3354,3068,3027,2926,1667$, and $1454 \mathrm{~cm}^{-1}$; HRMS-CI $\mathrm{m} / \mathrm{z} 190.1350\left[\mathrm{M}^{+}\right.$; calcd for $\mathrm{C}_{13} \mathrm{H}_{18} \mathrm{O}$ : 190.1358$]$.

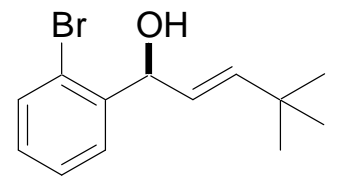

(S)-1-(2-Bromo-phenyl)-4,4-dimethyl-pent-2-en-1-ol (3). The product was prepared by General Procedure A using $50 \mathrm{mg}(0.28$ mmol) $\mathrm{Cy}_{2} \mathrm{BH}, 35 \mu \mathrm{L}$ (0.28 mmol) 3,3-dimethyl-1-butyne, $0.26 \mathrm{~mL}$ (0.53 mmol, 2.0 M in hexanes) $\mathrm{Et}_{2} \mathrm{Zn}, 2.4 \mathrm{mg}(0.01 \mathrm{mmol})(-)-$ $\mathrm{MIB}$, and $29 \mu \mathrm{L}(0.25 \mathrm{mmol}) o$-bromobenzaldehyde. The crude product was purified by column chromatography (5\% ethyl acetate in hexanes) to afford the title compound as a colorless oil in $76 \%$ yield $(51 \mathrm{mg}, 0.19 \mathrm{mmol}) . \quad[\alpha]_{\mathrm{D}}{ }^{20}=-31.8\left(c=0.33, \mathrm{CHCl}_{3}, 93 \%\right.$ ee); ${ }^{1} \mathrm{H}$ NMR $\left(\mathrm{CDCl}_{3}, 500 \mathrm{MHz}\right): \delta 1.02(\mathrm{~s}, 9 \mathrm{H}), 2.03(\mathrm{~s}, 1 \mathrm{H}), 5.46-5.53(\mathrm{~m}, 2 \mathrm{H}), 5.86$ $(\mathrm{d}, 1 \mathrm{H}, J=15.0 \mathrm{~Hz}), 7.13(\mathrm{t}, 1 \mathrm{H}, J=7.6 \mathrm{~Hz}), 7.33(\mathrm{t}, 1 \mathrm{H}, J=7.5 \mathrm{~Hz}), 7.52(\mathrm{~d}, 1 \mathrm{H}, J=$ $8.9 \mathrm{~Hz})$, and $7.54(\mathrm{~d}, 1 \mathrm{H}, J=8.2 \mathrm{~Hz}) \mathrm{ppm} ;{ }^{13} \mathrm{C}\left\{{ }^{1} \mathrm{H}\right\} \mathrm{NMR}\left(\mathrm{CDCl}_{3}, 125 \mathrm{MHz}\right): \delta 29.4$, $33.0,73.8,122.6,125.0,127.7,127.8,128.9,132.8,142.3$, and $144.3 \mathrm{ppm}$; IR (neat): $3353,3064,3013,2958,2865,1721,1661,1590,1568,1465,1439,1363$, and $1267 \mathrm{~cm}^{-1}$; HRMS-CI $m / z 251.0432$ [(M-OH $)^{+}$; calcd for $\mathrm{C}_{13} \mathrm{H}_{16} \mathrm{Br}$ : 251.0435].

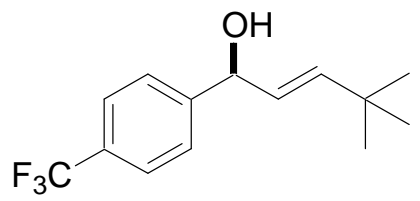

(S)-4,4-Dimethyl-1-(4-trifluoromethyl-phenyl)-pent-2-en-1ol (5). The product was prepared by General Procedure A using $98 \mathrm{mg}(0.55 \mathrm{mmol}) \mathrm{Cy}_{2} \mathrm{BH}, 68 \mu \mathrm{L}(0.55 \mathrm{mmol}) 3,3-$ dimethyl-1-butyne, $0.55 \mathrm{~mL}$ (1.1 mmol, $2.0 \mathrm{M}$ in hexanes) $\mathrm{Et}_{2} \mathrm{Zn}, \quad 4.8 \mathrm{mg} \quad(0.02 \mathrm{mmol}) \quad(-)-\mathrm{MIB}, \quad$ and $68 \mu \mathrm{L} \quad(0.50 \quad \mathrm{mmol}) \quad p-$ trifluoromethylbenzaldehyde. The crude product was purified by column chromatography (5\% ethyl acetate in hexanes) to afford the title compound as a white solid in $89 \%$ yield $(115 \mathrm{mg}, 0.45 \mathrm{mmol})$. m.p.: $30-32{ }^{\circ} \mathrm{C} ;[\alpha]_{\mathrm{D}}{ }^{20}=-10.0 \quad(c=0.27$, $\mathrm{CHCl}_{3}, 95 \%$ ee); ${ }^{1} \mathrm{H}$ NMR $\left(\mathrm{CDCl}_{3}, 500 \mathrm{MHz}\right): \delta 0.98(\mathrm{~s}, 9 \mathrm{H}), 2.03(\mathrm{br} \mathrm{s}, 1 \mathrm{H}), 5.15(\mathrm{~d}$, $1 \mathrm{H}, J=7.0 \mathrm{~Hz}), 5.47(\mathrm{dd}, 1 \mathrm{H}, J=15.5,7.2 \mathrm{~Hz}), 5.78(\mathrm{~d}, 1 \mathrm{H}, J=15.6 \mathrm{~Hz}), 7.43(\mathrm{~d}, 2 \mathrm{H}, J$ $=8.0 \mathrm{~Hz})$, and $7.55(\mathrm{~d}, 2 \mathrm{H}, J=8.0 \mathrm{~Hz}) \mathrm{ppm} ;{ }^{13} \mathrm{C}\left\{{ }^{1} \mathrm{H}\right\} \mathrm{NMR}\left(\mathrm{CDCl}_{3}, 125 \mathrm{MHz}\right): \delta 29.3$, $33.0,74.9,123.1,125.28,125.31,125.34,126.4,126.7,129.4,129.7,144.6$, and 147.3 
ppm; IR (film): 3335, 3025, 2962, 2905, 2868, 1661, 1620, 1477, 1463, 1414, 1364, and $1327 \mathrm{~cm}^{-1}$; HRMS-CI m/z 239.1247 [( $\left.\mathrm{M}-\mathrm{F}\right)^{+}$; calcd for $\left.\mathrm{C}_{14} \mathrm{H}_{17} \mathrm{~F}_{2} \mathrm{O}: 239.1247\right]$.<smiles>CCCC/C=C/C(O)c1ccc(C(F)(F)F)cc1</smiles>

trifluoromethylbenzaldehyde.

(S)-1-(4-Trifluoromethyl-phenyl)-hept-2-en-1-ol

(6). The product was prepared by General Procedure A using $98 \mathrm{mg}(0.55 \mathrm{mmol}) \mathrm{Cy}_{2} \mathrm{BH}, 64 \mu \mathrm{L}(0.55 \mathrm{mmol})$ 1-hexyne, $0.55 \mathrm{~mL}$ (1.1 mmol, 2.0 $\mathrm{M}$ in hexanes) $\mathrm{Et}_{2} \mathrm{Zn}, 4.8 \mathrm{mg}$ (0.02 mmol) (-)-MIB, and $68 \mu \mathrm{L}(0.50 \mathrm{mmol}) \quad p$ The crude product was purified by column chromatography (5\% ethyl acetate in hexanes) to afford the title compound as a colorless oily solid in $94 \%$ yield $(121 \mathrm{mg}, 0.47 \mathrm{mmol}) .[\alpha]_{\mathrm{D}}{ }^{20}=+69.3\left(c=0.88, \mathrm{CHCl}_{3}, 95 \%\right.$ ee $)$; ${ }^{1} \mathrm{H}$ NMR $\left(\mathrm{CDCl}_{3}, 500 \mathrm{MHz}\right): \delta 0.88(\mathrm{t}, 3 \mathrm{H}, J=7.1 \mathrm{~Hz}), 1.25-1.39(\mathrm{~m}, 4 \mathrm{H}), 1.61(\mathrm{br} \mathrm{s}$, $1 \mathrm{H}), 2.05(\mathrm{dt}, 2 \mathrm{H}, J=6.9,6.7 \mathrm{~Hz}), 5.19(\mathrm{~d}, 1 \mathrm{H}, J=7.0 \mathrm{~Hz}), 5.59(\mathrm{dd}, 1 \mathrm{H}, J=15.3,7.1$ $\mathrm{Hz}), 5.77(\mathrm{dt}, 1 \mathrm{H}, J=15.3,6.7 \mathrm{~Hz}), 7.46(\mathrm{~d}, 2 \mathrm{H}, J=8.0 \mathrm{~Hz})$, and $7.58(\mathrm{~d}, 2 \mathrm{H}, J=8.0 \mathrm{~Hz})$ ppm; ${ }^{13} \mathrm{C}\left\{{ }^{1} \mathrm{H}\right\}$ NMR $\left(\mathrm{CDCl}_{3}, 125 \mathrm{MHz}\right): \delta 13.9,22.2,31.3,31.8,74.7,125.3,125.4$, $126.4,129.4,129.7,131.7,133.9$, and $147.2 \mathrm{ppm}$; IR (neat): 3370, 3037, 2959, 2930, 2859, 2353, 1666, 1620, 1415, 1327, and $1165 \mathrm{~cm}^{-1}$; HRMS-ESI $\mathrm{m} / z 258.1239 \mathrm{CM}^{+}$; calcd for $\left.\mathrm{C}_{14} \mathrm{H}_{17} \mathrm{~F}_{3} \mathrm{O}: 258.1232\right]$.

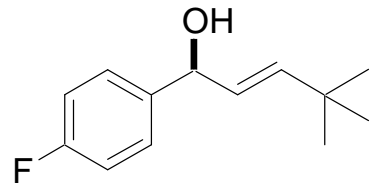

(S)-1-(4-Fluoro-phenyl)-4,4-dimethyl-pent-2-en-1-ol (7). The product was prepared by General Procedure A using $98 \mathrm{mg}(0.55$ mmol) $\mathrm{Cy}_{2} \mathrm{BH}, 68 \mu \mathrm{L}$ (0.55 mmol) 3,3-dimethyl-1-butyne, 0.55 $\mathrm{mL}\left(1.1 \mathrm{mmol}, 2.0 \mathrm{M}\right.$ in hexanes) $\mathrm{Et}_{2} \mathrm{Zn}, 4.8 \mathrm{mg}(0.02 \mathrm{mmol})$ (-)-MIB, and $54 \mu \mathrm{L}(0.50 \mathrm{mmol}) p$-fluorobenzaldehyde. The crude product was purified by column chromatography (5\% ethyl acetate in hexanes) to afford the title compound as a white solid in $82 \%$ yield $(85 \mathrm{mg}, 0.41 \mathrm{mmol})$. m.p.: $38-40{ }^{\circ} \mathrm{C} ;[\alpha]_{\mathrm{D}}{ }^{20}=+21.3(c=1.2$, $\mathrm{CHCl}_{3}, 93 \%$ ee); ${ }^{1} \mathrm{H} \mathrm{NMR}\left(\mathrm{CDCl}_{3}, 500 \mathrm{MHz}\right): \delta 1.01$ (s, 9H), 1.84 (br s, $\left.1 \mathrm{H}\right), 5.13(\mathrm{~d}$, $1 \mathrm{H}, J=6.9 \mathrm{~Hz}), 5.52(\mathrm{dd}, 1 \mathrm{H}, J=15.6,6.9 \mathrm{~Hz}), 5.77(\mathrm{~d}, 1 \mathrm{H}, J=15.6 \mathrm{~Hz}), 7.01(\mathrm{t}, 2 \mathrm{H}, J$ $=8.7 \mathrm{~Hz})$, and $7.31(\mathrm{dd}, 2 \mathrm{H}, J=8.3,5.6 \mathrm{~Hz}) \mathrm{ppm} ;{ }^{13} \mathrm{C}\left\{{ }^{1} \mathrm{H}\right\} \mathrm{NMR}\left(\mathrm{CDCl}_{3}, 125 \mathrm{MHz}\right): \delta$ 29.4, 32.9, 74.7, 115.1, 115.3, 127.1, 127.77, 127.83, 139.18, 139.20, 143.7, 161.1, and 163.1 ppm; IR (film): 3344, 3025, 2959, 2866, 1725, 1661, 1604, 1509, 1463, 1363, 1294, 1225, and $1156 \mathrm{~cm}^{-1}$; HRMS-CI m/z 208.1267 $\left[\mathrm{M}^{+}\right.$; calcd for $\mathrm{C}_{13} \mathrm{H}_{17} \mathrm{FO}$ : 208.1263].

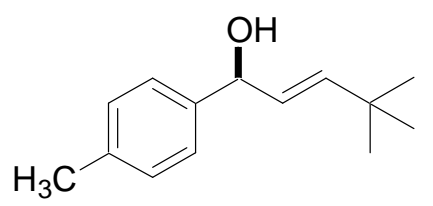

(S)-4,4-Dimethyl-1-p-tolyl-pent-2-en-1ol (8). The product was prepared by General Procedure A using $98 \mathrm{mg}(0.55$ mmol) $\mathrm{Cy}_{2} \mathrm{BH}, 68 \mu \mathrm{L}(0.55 \mathrm{mmol})$ 3,3-dimethyl-1-butyne, $0.55 \mathrm{~mL}$ (1.1 mmol, 2.0 $\mathrm{M}$ in hexanes) $\mathrm{Et}_{2} \mathrm{Zn}, 4.8 \mathrm{mg}(0.02$ mmol) (-)-MIB, and $59 \mu \mathrm{L}(0.50 \mathrm{mmol}) p$-tolualdehyde. The crude product was purified by column chromatography (5\% ethyl acetate in hexanes) to afford the title compound as a white solid in $86 \%$ yield ( $88 \mathrm{mg}, 0.43 \mathrm{mmol})$. m.p.: $38-40$ ${ }^{\circ} \mathrm{C} ;[\alpha]_{\mathrm{D}}{ }^{20}=+52.7\left(c=0.97, \mathrm{CHCl}_{3}, 95 \%\right.$ ee $) ;{ }^{1} \mathrm{H} \mathrm{NMR}\left(\mathrm{CDCl}_{3}, 500 \mathrm{MHz}\right): \delta 0.95(\mathrm{~s}$, $9 \mathrm{H}), 1.83$ (br s, 1H), 2.26 (s, 3H), $5.03(\mathrm{~d}, 1 \mathrm{H}, J=6.7 \mathrm{~Hz}), 5.47$ (dd, 1H, $J=15.6,6.8$ $\mathrm{Hz}), 5.70(\mathrm{~d}, 1 \mathrm{H}, J=15.6 \mathrm{~Hz}), 7.07(\mathrm{~d}, 2 \mathrm{H}, J=7.7 \mathrm{~Hz})$, and $7.17(\mathrm{~d}, 2 \mathrm{H}, J=7.9 \mathrm{~Hz})$ ppm; ${ }^{13} \mathrm{C}\left\{{ }^{1} \mathrm{H}\right\} \mathrm{NMR}\left(\mathrm{CDCl}_{3}, 125 \mathrm{MHz}\right): \delta 21.1,29.4,32.8,75.2,126.1,127.2,129.1$, 137.1, 140.6, and 143.2 ppm; IR (film): 3346, 3023, 2959, 2865, 2361, 2334, 1655, 
$1613,1513,1478,1461,1416,1388,1362$, and $1268 \mathrm{~cm}^{-1}$; HRMS-CI $\mathrm{m} / 2.204 .1509\left[\mathrm{M}^{+}\right.$; calcd for $\mathrm{C}_{14} \mathrm{H}_{20} \mathrm{O}$ : 204.1514].

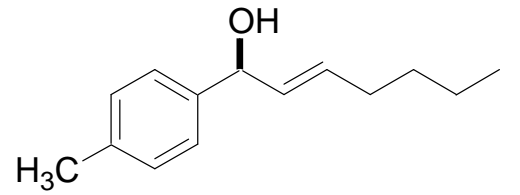

(S)-1-p-Tolyl-hept-2-en-1-ol (9). The product was prepared by General Procedure A using $98 \mathrm{mg}(0.55$ mmol) $\mathrm{Cy}_{2} \mathrm{BH}, 64 \mu \mathrm{L}(0.55 \mathrm{mmol})$ 1-hexyne, $0.55 \mathrm{~mL}$ (1.1 mmol, 2.0 M in hexanes) Et $2 \mathrm{Zn}, 4.8 \mathrm{mg}(0.02 \mathrm{mmol})$ (-)-MIB, and $59 \mu \mathrm{L}(0.50 \mathrm{mmol}) p$-tolualdehyde. The crude product was purified by column chromatography (5\% ethyl acetate in hexanes) to afford the title compound as a colorless oil in $87 \%$ yield $(89 \mathrm{mg}, 0.44 \mathrm{mmol}) .[\alpha]_{\mathrm{D}}^{20}=+43.1\left(c=1.3, \mathrm{CHCl}_{3}, 95 \%\right.$ ee $)$; ${ }^{1} \mathrm{H}$ NMR $\left(\mathrm{CDCl}_{3}, 500 \mathrm{MHz}\right): \quad \delta 0.81(\mathrm{t}, 3 \mathrm{H}, J=7.1 \mathrm{~Hz}), 1.20-1.32(\mathrm{~m}, 4 \mathrm{H}), 1.81(\mathrm{br} \mathrm{s}$, $1 \mathrm{H}), 1.97(\mathrm{dt}, 2 \mathrm{H}, J=7.0,6.6 \mathrm{~Hz}), 2.26(\mathrm{~s}, 3 \mathrm{H}), 5.04(\mathrm{~d}, 1 \mathrm{H}, J=6.5 \mathrm{~Hz}), 5.57(\mathrm{dd}, 1 \mathrm{H}, J$ $=15.3,6.6 \mathrm{~Hz}), 5.66(\mathrm{dt}, 1 \mathrm{H}, J=15.3,6.5 \mathrm{~Hz}), 7.07(\mathrm{~d}, 2 \mathrm{H}, J=7.8 \mathrm{~Hz})$, and $7.17(\mathrm{~d}, 2 \mathrm{H}$, $J=7.8 \mathrm{~Hz}) \mathrm{ppm} ;{ }^{13} \mathrm{C}\left\{{ }^{1} \mathrm{H}\right\} \mathrm{NMR}\left(\mathrm{CDCl}_{3}, 125 \mathrm{MHz}\right): \delta 13.9,21.1,22.2,31.2,31.9,75.1$, 126.1, 129.1, 132.3, 132.5, 137.1, and 140.5 ppm; IR (neat): 3346, 3017, 2957, 2925, $2858,1663,1613,1513,1456,1377$, and $1198 \mathrm{~cm}^{-1}$; HRMS-CI $\mathrm{m} / z$ 203.1440 [(M - H $)^{+}$; calcd for $\left.\mathrm{C}_{14} \mathrm{H}_{19} \mathrm{O}: 203.1436\right]$.

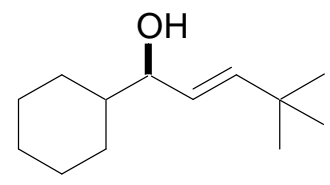

(S)-1-Cyclohexyl-4,4-dimethyl-pent-2-en-1-ol (10). The product was prepared by General Procedure A using $98 \mathrm{mg}(0.55$ mmol) $\mathrm{Cy}_{2} \mathrm{BH}, 68 \mu \mathrm{L}$ (0.55 mmol) 3,3-dimethyl-1-butyne, $0.55 \mathrm{~mL}$ $\left(1.1 \mathrm{mmol}, 2.0 \mathrm{M}\right.$ in hexanes) $\mathrm{Et}_{2} \mathrm{Zn}, 4.8 \mathrm{mg}(0.02 \mathrm{mmol})(-)-\mathrm{MIB}$, and $61 \mu \mathrm{L}(0.50 \mathrm{mmol})$ cyclohexane- carboxaldehyde. The crude product was purified by column chromatography (5\% ethyl acetate in hexanes) to afford the title compound as a colorless oil in $80 \%$ yield $(78 \mathrm{mg}, 0.40 \mathrm{mmol}) .[\alpha]_{\mathrm{D}}{ }^{20}=-27.7\left(c=0.13, \mathrm{CHCl}_{3}, 96 \%\right.$ ee); ${ }^{1} \mathrm{H}$ NMR $\left(\mathrm{CDCl}_{3}, 500 \mathrm{MHz}\right): \delta 0.92-0.97(\mathrm{~m}, 2 \mathrm{H}), 1.01(\mathrm{~s}, 9 \mathrm{H}), 1.11-1.26(\mathrm{~m}, 4 \mathrm{H})$, $1.41(\mathrm{~m}, 1 \mathrm{H}), 1.64(\mathrm{~m}, 2 \mathrm{H}), 1.73(\mathrm{~m}, 2 \mathrm{H}), 1.86(\mathrm{br} \mathrm{d}, 1 \mathrm{H}, J=12.6 \mathrm{~Hz}), 3.75(\mathrm{t}, 1 \mathrm{H}, J=$ $7.0 \mathrm{~Hz}), 5.35(\mathrm{dd}, 1 \mathrm{H}, J=15.6,7.5 \mathrm{~Hz})$, and $5.62(\mathrm{~d}, 1 \mathrm{H}, J=15.7 \mathrm{~Hz}) \mathrm{ppm} ;{ }^{13} \mathrm{C}\left\{{ }^{1} \mathrm{H}\right\}$ NMR $\left(\mathrm{CDCl}_{3}, 125 \mathrm{MHz}\right): \delta 26.1,26.2,26.6,28.76,28.84,29.6,32.9,43.8,77.9,126.2$, and $143.9 \mathrm{ppm}$; IR (neat): 3351, 2953, 2926, 2853, 1655, 1473, 1450, 1387, 1362, 1305, 1260, and $1204 \mathrm{~cm}^{-1}$; HRMS-CI $\mathrm{m} / z$ 195.1743 [(M-H $)^{+}$; calcd for $\left.\mathrm{C}_{13} \mathrm{H}_{23} \mathrm{O}: 195.1749\right]$.

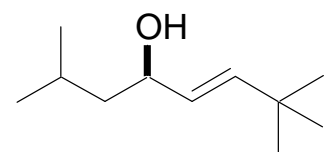

(S)-2,7,7-Trimethyl-oct-5-en-4-ol (11). The product was prepared by General Procedure A using $196 \mathrm{mg}(1.1 \mathrm{mmol}) \mathrm{Cy}_{2} \mathrm{BH}, 137 \mu \mathrm{L}$ (1.1 mmol) 3,3-dimethyl-1-butyne, $1.1 \mathrm{~mL}(2.1 \mathrm{mmol}, 2.0 \mathrm{M}$ in hexanes) $\mathrm{Et}_{2} \mathrm{Zn}, 9.6 \mathrm{mg}(0.04 \mathrm{mmol})(-)-\mathrm{MIB}$, and $107 \mu \mathrm{L}(1.0$ $\mathrm{mmol}$ ) isovaleraldehyde. The crude product was purified by column chromatography (5\% ethyl acetate in hexanes) to afford the title compound as a colorless oil in $86 \%$ yield $(146 \mathrm{mg}, 0.86 \mathrm{mmol}) .[\alpha]_{\mathrm{D}}{ }^{20}=+16.7\left(c=3.0, \mathrm{CHCl}_{3}, 85 \%\right.$ ee $) ;{ }^{1} \mathrm{H} \mathrm{NMR}\left(\mathrm{CDCl}_{3}, 500\right.$ $\mathrm{MHz}): \delta 0.88(\mathrm{t}, 6 \mathrm{H}, J=7.0 \mathrm{~Hz}), 0.97(\mathrm{~s}, 9 \mathrm{H}), 1.26(\mathrm{~m}, 1 \mathrm{H}), 1.43(\mathrm{~m}, 1 \mathrm{H}), 1.50(\mathrm{br} \mathrm{s}$, $1 \mathrm{H}), 1.65(\mathrm{~m}, 1 \mathrm{H}), 4.07(\mathrm{dt}, 1 \mathrm{H}, J=7.0,6.8 \mathrm{~Hz}), 5.31(\mathrm{dd}, 1 \mathrm{H}, J=15.6,7.2 \mathrm{~Hz})$, and $5.61(\mathrm{~d}, 1 \mathrm{H}, J=15.7 \mathrm{~Hz}) \mathrm{ppm} ;{ }^{13} \mathrm{C}\left\{{ }^{1} \mathrm{H}\right\} \mathrm{NMR}\left(\mathrm{CDCl}_{3}, 125 \mathrm{MHz}\right): \delta 22.5,22.9,24.6$, 29.5, 32.5, 46.6, 71.5, 128.1, and 142.6 ppm; IR (neat): 3334, 3013, 2956, 2869, 2354, 2320, 1662, 1466, 1386, 1363, 1308, 1268, 1220, and $1202 \mathrm{~cm}^{-1}$; HRMS-CI $\mathrm{m} / \mathrm{z}$ $170.1666\left[\mathrm{M}^{+}\right.$; calcd for $\left.\mathrm{C}_{11} \mathrm{H}_{22} \mathrm{O}: 170.1671\right]$. 


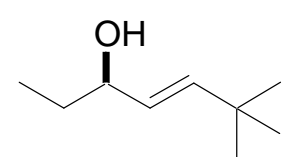

(S)-6,6-Dimethyl-hept-4-en-3-ol (12). The product was prepared by General Procedure A using $196 \mathrm{mg}(1.1 \mathrm{mmol}) \mathrm{Cy}_{2} \mathrm{BH}, 137 \mu \mathrm{L}(1.1$ mmol) 3,3-dimethyl-1-butyne, $1.1 \mathrm{~mL}$ (2.1 mmol, $2.0 \mathrm{M}$ in hexanes) $\mathrm{Et}_{2} \mathrm{Zn}, 9.6 \mathrm{mg}(0.04 \mathrm{mmol})(-)-\mathrm{MIB}$, and $72 \mu \mathrm{L}(1.0 \mathrm{mmol})$ propanal. The crude product was purified by column chromatography (5\% ethyl acetate in hexanes) to afford the title compound as a colorless oil in $68 \%$ yield $(96 \mathrm{mg}, 0.68 \mathrm{mmol}) .[\alpha]_{\mathrm{D}}{ }^{20}=$ $-60.3\left(c=0.63, \mathrm{CHCl}_{3}, 77 \%\right.$ ee); ${ }^{1} \mathrm{H} \mathrm{NMR}\left(\mathrm{CDCl}_{3}, 500 \mathrm{MHz}\right): \delta 0.86(\mathrm{t}, 3 \mathrm{H}, J=7.4$ $\mathrm{Hz}), 0.98(\mathrm{~s}, 9 \mathrm{H}), 1.26(\mathrm{br} \mathrm{s}, 1 \mathrm{H}), 1.44-1.59(\mathrm{~m}, 2 \mathrm{H}), 3.93(\mathrm{dt}, 1 \mathrm{H}, J=6.6,6.6 \mathrm{~Hz}), 5.31$ $(\mathrm{dd}, 1 \mathrm{H}, J=15.7,7.1 \mathrm{~Hz})$, and $5.62(\mathrm{~d}, 1 \mathrm{H}, J=15.7 \mathrm{~Hz}) \mathrm{ppm} ;{ }^{13} \mathrm{C}\left\{{ }^{1} \mathrm{H}\right\} \mathrm{NMR}\left(\mathrm{CDCl}_{3}\right.$, $125 \mathrm{MHz}$ ): $\delta 9.7,22.6,29.5,30.2,74.7,127.4$, and $143.2 \mathrm{ppm}$; IR (neat): 3360, 3025, 2960, 2866, 1660, 1478, 1463, 1414, 1384, 1363, 1331, and $1262 \mathrm{~cm}^{-1}$; HRMS-CI $\mathrm{m} / \mathrm{z}$ $141.1274\left[(\mathrm{M}-\mathrm{H})^{+}\right.$; calcd for $\left.\mathrm{C}_{9} \mathrm{H}_{17} \mathrm{O}: 141.1279\right]$.

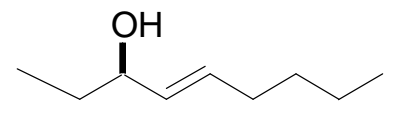

(S)-Non-4-en-3-ol (13). The product was prepared by General Procedure A using $50 \mathrm{mg}(0.28 \mathrm{mmol}) \mathrm{Cy}_{2} \mathrm{BH}, 32 \mu \mathrm{L}(0.28$ $\mathrm{mmol})$ 1-hexyne, $0.39 \mathrm{~mL}\left(0.78 \mathrm{mmol}, 2.0 \mathrm{M}\right.$ in hexanes) $\mathrm{Et}_{2} \mathrm{Zn}$, $2.4 \mathrm{mg}(0.01 \mathrm{mmol})(-)-\mathrm{MIB}$, and $18 \mu \mathrm{L}(0.25 \mathrm{mmol})$ propanal. The crude product was purified by column chromatography (5\% ethyl acetate in hexanes) to afford the title compound as a colorless oil in $74 \%$ yield $(26 \mathrm{mg}, 0.18 \mathrm{mmol}) .[\alpha]_{\mathrm{D}}{ }^{20}=+58.5(c=0.97$, $\mathrm{CHCl}_{3}, 79 \%$ ee); ${ }^{1} \mathrm{H} \mathrm{NMR}\left(\mathrm{CDCl}_{3}, 500 \mathrm{MHz}\right): \delta 0.87(\mathrm{~m}, 6 \mathrm{H}), 1.22-1.36(\mathrm{~m}, 4 \mathrm{H}), 1.39$ (br s, $1 \mathrm{H}), 1.43-1.57(\mathrm{~m}, 2 \mathrm{H}), 2.00(\mathrm{dt}, 2 \mathrm{H}, J=7.0,6.6 \mathrm{~Hz}), 3.94(\mathrm{dt}, 1 \mathrm{H}, J=6.6,6.6$ $\mathrm{Hz}), 5.41(\mathrm{dd}, 1 \mathrm{H}, J=15.3,7.0 \mathrm{~Hz})$, and $5.60(\mathrm{dt}, 1 \mathrm{H}, J=15.3,6.7 \mathrm{~Hz}) \mathrm{ppm} ;{ }^{13} \mathrm{C}\left\{{ }^{1} \mathrm{H}\right\}$ NMR $\left(\mathrm{CDCl}_{3}, 125 \mathrm{MHz}\right): \delta 9.7,13.9,22.2,30.2,31.4,31.9,74.6,132.4$, and $132.7 \mathrm{ppm}$; IR (neat): 3421, 2955, 2921, 2854, 2361, 2337, 1725, 1663, 1601, 1455, 1405, and 1259 $\mathrm{cm}^{-1}$; HRMS-CI $\mathrm{m} / z$ 141.1273 [(M - H $)^{+}$; calcd for $\mathrm{C}_{9} \mathrm{H}_{17} \mathrm{O}$ : 141.1279].

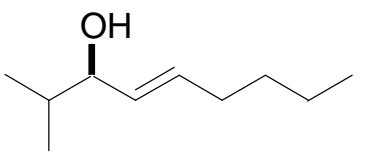

(S)-2-Methyl-non-4-en-3-ol (14). The product was prepared by General Procedure A using $98 \mathrm{mg}(0.55 \mathrm{mmol}) \mathrm{Cy}_{2} \mathrm{BH}, 64 \mu \mathrm{L}$ (0.55 mmol) 1-hexyne, $0.55 \mathrm{~mL}$ (1.1 mmol, $2.0 \mathrm{M}$ in hexanes) $\mathrm{Et}_{2} \mathrm{Zn}, 4.8 \mathrm{mg}(0.02 \mathrm{mmol})(-)-\mathrm{MIB}$, and $45 \mu \mathrm{L}(0.50 \mathrm{mmol})$ isobutyraldehyde. The crude product was purified by column chromatography (5\% ethyl acetate in hexanes) to afford the title compound as a colorless oil in $88 \%$ yield $(69 \mathrm{mg}$, $0.44 \mathrm{mmol}) .[\alpha]_{\mathrm{D}}{ }^{20}=-12.5\left(c=1.3, \mathrm{CHCl}_{3}, 92 \%\right.$ ee $) ;{ }^{1} \mathrm{H} \mathrm{NMR}\left(\mathrm{CDCl}_{3}, 500 \mathrm{MHz}\right): \delta$ $0.82(\mathrm{~d}, 3 \mathrm{H}, J=6.7 \mathrm{~Hz}), 0.84(\mathrm{t}, 3 \mathrm{H}, J=7.2 \mathrm{~Hz}), 0.87(\mathrm{~d}, 3 \mathrm{H}, J=6.7 \mathrm{~Hz}), 1.23-1.32(\mathrm{~m}$, $4 \mathrm{H}), 1.48(\mathrm{br} \mathrm{s}, 1 \mathrm{H}), 1.64(\mathrm{~m}, 1 \mathrm{H}), 1.99(\mathrm{dt}, 2 \mathrm{H}, J=6.8,6.5 \mathrm{~Hz}), 3.72(\mathrm{t}, 1 \mathrm{H}, J=6.5 \mathrm{~Hz})$, $5.40(\mathrm{dd}, 1 \mathrm{H}, J=15.3,7.2 \mathrm{~Hz})$, and $5.57(\mathrm{dt}, 1 \mathrm{H}, J=15.3,6.7 \mathrm{~Hz}) \mathrm{ppm} ;{ }^{13} \mathrm{C}\left\{{ }^{1} \mathrm{H}\right\} \mathrm{NMR}$ $\left(\mathrm{CDCl}_{3}, 125 \mathrm{MHz}\right): \delta$ 13.9, 18.1, 18.2, 22.2, 31.4, 32.0, 33.8, 78.3, 131.1, and 133.2 ppm; IR (neat): 3221, 2953, 2925, 2862, 1488, 1466, 1381, 1331, 1260, and $1194 \mathrm{~cm}^{-1}$; LRMS-CI $m / z 139.14$ [(M-OH $)^{+}$; calcd for $\mathrm{C}_{10} \mathrm{H}_{19}$ : 139.15].

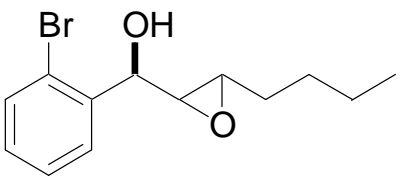

(2-Bromo-phenyl)-(3-butyl-oxiranyl)-methanol (18). General Procedure B. A $10 \mathrm{~mL}$ Schlenk flask (A) was charged with $\mathrm{Cy}_{2} \mathrm{BH}(98 \mathrm{mg}, 0.55 \mathrm{mmol})$, prepared according to Oppolzer's procedure, ${ }^{1}$ and $1.2 \mathrm{~mL}$ of hexanes. 1-Hexyne $(64 \mu \mathrm{L}, 0.55 \mathrm{mmol})$ was added dropwise, and the homogenous reaction mixture was stirred for $30 \mathrm{~min}$ at room temp. After cooling the reaction flask to $-10{ }^{\circ} \mathrm{C},(-)$-MIB $(4.8$ 
$\mathrm{mg}, \quad 0.02 \mathrm{mmol})$ was added, followed by $\mathrm{Et}_{2} \mathrm{Zn}(0.78 \mathrm{~mL}, 2.0 \mathrm{M})$, then $o$ bromobenzaldehyde $(58 \mu \mathrm{L}, 0.50 \mathrm{mmol})$ dropwise. The reaction was stirred at $-10{ }^{\circ} \mathrm{C}$ for $4 \mathrm{~h}$ until vinyl addition was complete by TLC. The reaction flask was then capped with a balloon of oxygen and allowed to stir at $-10{ }^{\circ} \mathrm{C}$ for $1 \mathrm{~h}$. In a separate $10 \mathrm{~mL}$ Schlenk flask (B), $1 \mathrm{~mL}$ of dichloromethane, $\operatorname{Ti}(\mathrm{OiPr})_{4}(100 \mu \mathrm{L}, 1.0 \mathrm{M}$ in hexanes), and (+)-DIPT $(21 \mu \mathrm{L}, 0.10 \mathrm{mmol})$ were combined. After stirring at room temp for $45 \mathrm{~min}$, flask B was evacuated for $1 \mathrm{~h}$ to strip off the solvent. The contents of flask B were then redissolved in $1 \mathrm{~mL}$ of dichloromethane and stripped again for $1 \mathrm{~h}$. Finally, the contents of flask B were taken up in $1 \mathrm{~mL}$ of dichloromethane and transferred to reaction flask A. The reaction continued to stir at $-10{ }^{\circ} \mathrm{C}$ under an $\mathrm{O}_{2}$ atmosphere for $18 \mathrm{~h}$. It was then quenched with $2 \mathrm{~mL} 15 \%$ tartaric acid solution and allowed to stir for $45 \mathrm{~min}$ before the organic and aqueous layers were separated, and the aqueous layer was extracted with hexanes $(3 \times 5 \mathrm{~mL})$. The combined organic layers were then washed with $5 \mathrm{~mL} \mathrm{H}_{2} \mathrm{O}$ and dried over $\mathrm{MgSO}_{4}$. The filtrate was concentrated in vacuo and the residue was chromatographed on silica (5\% ethyl acetate in hexanes) to afford the title compound in $77 \%$ yield $(110 \mathrm{mg}, 0.39 \mathrm{mmol})$. threo-diastereomer: Colorless oil. $[\alpha]_{\mathrm{D}}{ }^{20}=-40.5(c=$ $\left.0.39, \mathrm{CHCl}_{3}\right) ;{ }^{1} \mathrm{H} \mathrm{NMR}\left(\mathrm{CDCl}_{3}, 500 \mathrm{MHz}\right): \delta 0.87(\mathrm{t}, 3 \mathrm{H}, J=7.1 \mathrm{~Hz}), 1.27-1.44(\mathrm{~m}$, $4 \mathrm{H}), 1.53-1.61(\mathrm{~m}, 2 \mathrm{H}), 2.51(\mathrm{~d}, 1 \mathrm{H}, J=5.4 \mathrm{~Hz}), 2.98(\mathrm{dd}, 1 \mathrm{H}, J=4.5,2.1 \mathrm{~Hz}), 3.24(\mathrm{dt}$, $1 \mathrm{H}, J=5.6,2.0 \mathrm{~Hz}), 5.02(\mathrm{t}, 1 \mathrm{H}, J=4.9 \mathrm{~Hz}), 7.18(\mathrm{t}, 1 \mathrm{H}, J=7.6 \mathrm{~Hz}), 7.37(\mathrm{t}, 1 \mathrm{H}, J=7.5$ $\mathrm{Hz}), 7.56(\mathrm{~d}, 1 \mathrm{H}, J=7.9 \mathrm{~Hz})$, and $7.61(\mathrm{~d}, 1 \mathrm{H}, J=7.7 \mathrm{~Hz}) \mathrm{ppm} ;{ }^{13} \mathrm{C}\left\{{ }^{1} \mathrm{H}\right\} \mathrm{NMR}\left(\mathrm{CDCl}_{3}\right.$, $125 \mathrm{MHz}): \delta 13.9,22.4,27.9,31.1,57.7,61.1,71.6,122.1,127.9,128.0,129.5,132.8$, and 139.8 ppm; IR (neat): 3424, 3063, 2962, 2930, 2861, 1562, 1468, 1434, 1383, and $1260 \mathrm{~cm}^{-1}$; HRMS-CI m/z 285.0496 $\left[\mathrm{MH}^{+}\right.$; calcd for $\mathrm{C}_{13} \mathrm{H}_{18} \mathrm{BrO}_{2}:$ 285.0490]. erythrodiastereomer: Colorless oil. $[\alpha]_{\mathrm{D}}{ }^{20}=-55.9\left(c=1.2, \mathrm{CHCl}_{3}\right) ;{ }^{1} \mathrm{H} \mathrm{NMR}\left(\mathrm{CDCl}_{3}, 500\right.$ $\mathrm{MHz}): \delta 0.82(\mathrm{t}, 3 \mathrm{H}, J=7.1 \mathrm{~Hz}), 1.20-1.33(\mathrm{~m}, 4 \mathrm{H}), 1.44(\mathrm{~m}, 1 \mathrm{H}), 1.55(\mathrm{~m}, 1 \mathrm{H}), 2.51(\mathrm{~s}$, $1 \mathrm{H}), 3.03(\mathrm{dt}, 1 \mathrm{H}, J=5.6,1.8 \mathrm{~Hz}), 3.20(\mathrm{br} \mathrm{t}, 1 \mathrm{H}), 5.37(\mathrm{br} \mathrm{s}, 1 \mathrm{H}), 7.17(\mathrm{t}, 1 \mathrm{H}, J=7.1$ $\mathrm{Hz}), 7.34(\mathrm{t}, 1 \mathrm{H}, J=7.5 \mathrm{~Hz}), 7.50(\mathrm{~d}, 1 \mathrm{H}, J=7.6 \mathrm{~Hz})$, and $7.54(\mathrm{~d}, 1 \mathrm{H}, J=8.0 \mathrm{~Hz}) \mathrm{ppm}$; ${ }^{13} \mathrm{C}\left\{{ }^{1} \mathrm{H}\right\} \mathrm{NMR}\left(\mathrm{CDCl}_{3}, 125 \mathrm{MHz}\right): \delta 13.8,22.2,27.9,31.0,54.8,59.6,69.1,122.1$, 127.7, 127.9, 129.4, 132.6, and 138.8 ppm; IR (neat): 3434, 3072, 2957, 2931, 2859, 1590, 1568, 1468, 1438, and $1378 \mathrm{~cm}^{-1}$; HRMS-CI $\mathrm{m} / z .285 .0496\left[\mathrm{MH}^{+}\right.$; calcd for $\mathrm{C}_{13} \mathrm{H}_{18} \mathrm{BrO}_{2}$ : 285.0490].

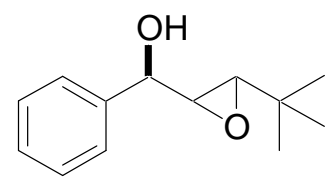

(3-tert-Butyl-oxiranyl)-phenyl-methanol (15). The product was prepared by General Procedure B using $50 \mathrm{mg}(0.28 \mathrm{mmol}) \mathrm{Cy}_{2} \mathrm{BH}$, $35 \mu \mathrm{L}(0.28 \mathrm{mmol}) 3,3$-dimethyl-1-butyne, $0.39 \mathrm{~mL}(0.78 \mathrm{mmol}$, 2.0 $\mathrm{M}$ in hexanes) $\mathrm{Et}_{2} \mathrm{Zn}, 2.4 \mathrm{mg}(0.01 \mathrm{mmol})(-)-\mathrm{MIB}, 25 \mu \mathrm{L}(0.25$ mmol) benzaldehyde, $50 \mu \mathrm{L}(0.05 \mathrm{mmol}, 1.0 \mathrm{M}$ in hexanes $) \mathrm{Ti}(\mathrm{O} i \mathrm{Pr})_{4}$, and $10.6 \mu \mathrm{L}(0.05$ mmol) (+)-DIPT. The crude product was purified by column chromatography (5\% ethyl acetate in hexanes) to afford the title compound in $77 \%$ yield $(40 \mathrm{mg}, 0.19 \mathrm{mmol})$. threodiastereomer: White solid. m.p.: $70-73{ }^{\circ} \mathrm{C} .[\alpha]_{\mathrm{D}}{ }^{20}=-25.6\left(c=0.16, \mathrm{CHCl}_{3}\right) ;{ }^{1} \mathrm{H} \mathrm{NMR}$ $\left(\mathrm{CDCl}_{3}, 500 \mathrm{MHz}\right): \delta 0.83(\mathrm{~s}, 9 \mathrm{H}), 2.32(\mathrm{br} \mathrm{s}, 1 \mathrm{H}), 2.82(\mathrm{~d}, 1 \mathrm{H}, J=1.6 \mathrm{~Hz}), 3.01(\mathrm{dd}$, $1 \mathrm{H}, J=5.3,1.9 \mathrm{~Hz}), 4.46(\mathrm{t}, 1 \mathrm{H}, J=4.4 \mathrm{~Hz}), 7.26(\mathrm{t}, 1 \mathrm{H}, J=6.9 \mathrm{~Hz})$, and 7.31-7.36 (m, 4H) ppm; ${ }^{13} \mathrm{C}\left\{{ }^{1} \mathrm{H}\right\} \mathrm{NMR}\left(\mathrm{CDCl}_{3}, 125 \mathrm{MHz}\right): \delta 25.7,30.6,59.3,65.1,74.2,126.0,128.0$, 128.6, and 140.5 ppm; IR (film): 3413, 3049, 3025, 2955, 2919, 2861, 2355, 1719, 1602, $1478,1455,1390,1360,1273,1237$, and $1196 \mathrm{~cm}^{-1}$; HRMS-CI $\mathrm{m} / z .189 .1288$ [(M - 
$\mathrm{OH})^{+}$; calcd for $\mathrm{C}_{13} \mathrm{H}_{17} \mathrm{O}$ : 189.1279]. erythro-diastereomer: White solid. m.p.: 57-59 ${ }^{\circ} \mathrm{C} ;[\alpha]_{\mathrm{D}}{ }^{20}=-27.1\left(c=0.31, \mathrm{CHCl}_{3}\right) ;{ }^{1} \mathrm{H} \mathrm{NMR}\left(\mathrm{CDCl}_{3}, 500 \mathrm{MHz}\right): \delta 0.82(\mathrm{~s}, 9 \mathrm{H}), 2.20$ (br s, $1 \mathrm{H}), 2.96(\mathrm{~d}, 1 \mathrm{H}, J=2.0 \mathrm{~Hz}), 3.01(\mathrm{t}, 1 \mathrm{H}, J=2.7 \mathrm{~Hz}), 4.82(\mathrm{br} \mathrm{s}, 1 \mathrm{H})$, and 7.26$7.32(\mathrm{~m}, 5 \mathrm{H}) \mathrm{ppm} ;{ }^{13} \mathrm{C}\left\{{ }^{1} \mathrm{H}\right\}$ NMR $\left(\mathrm{CDCl}_{3}, 125 \mathrm{MHz}\right): \delta 25.8,30.4,58.3,62.8,71.2$, 126.5, 128.3, 128.6, and $139.8 \mathrm{ppm}$; IR (film): 3413, 3037, 2955, 2931, 2861, 1719, $1643,1602,1449,1367$, and $1327 \mathrm{~cm}^{-1}$; HRMS-CI $\mathrm{m} / \mathrm{z} 189.1288\left[(\mathrm{M}-\mathrm{OH})^{+}\right.$; calcd for $\left.\mathrm{C}_{13} \mathrm{H}_{17} \mathrm{O}: 189.1279\right]$.

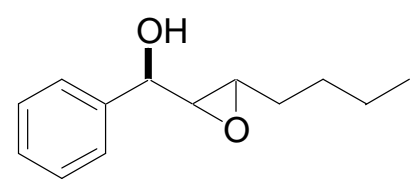

(3-Butyl-oxiranyl)-phenyl-methanol (16). The product was prepared by General Procedure B using $50 \mathrm{mg}(0.28 \mathrm{mmol})$ $\mathrm{Cy}_{2} \mathrm{BH}, 32 \mu \mathrm{L}(0.28 \mathrm{mmol})$ 1-hexyne, $0.39 \mathrm{~mL}(0.78 \mathrm{mmol}$, $2.0 \mathrm{M}$ in hexanes) $\mathrm{Et}_{2} \mathrm{Zn}, 2.4 \mathrm{mg}(0.01 \mathrm{mmol})(-)-\mathrm{MIB}, 25 \mu \mathrm{L}$ $(0.25 \mathrm{mmol})$ benzaldehyde, $50 \mu \mathrm{L}(0.05 \mathrm{mmol}, 1.0 \mathrm{M}$ in hexanes $) \mathrm{Ti}(\mathrm{O} i \mathrm{Pr})_{4}$, and $10.6 \mu \mathrm{L}$ $(0.05 \mathrm{mmol})(+)$-DIPT. The crude product was purified by column chromatography $(5 \%$ ethyl acetate in hexanes) to afford the title compound in $83 \%$ yield ( $43 \mathrm{mg}, 0.21 \mathrm{mmol})$. threo-diastereomer: Colorless oil. $[\alpha]_{\mathrm{D}}{ }^{20}=-12.9\left(c=2.5, \mathrm{CHCl}_{3}\right) ;{ }^{1} \mathrm{H} \mathrm{NMR}\left(\mathrm{CDCl}_{3}\right.$, $500 \mathrm{MHz}): \delta 0.83(\mathrm{t}, 3 \mathrm{H}, J=7.1 \mathrm{~Hz}), 1.22-1.41(\mathrm{~m}, 4 \mathrm{H}), 1.46-1.56(\mathrm{~m}, 2 \mathrm{H}), 2.31(\mathrm{br} \mathrm{s}$, $1 \mathrm{H}), 2.93(\mathrm{dt}, 1 \mathrm{H}, J=5.2,2.0 \mathrm{~Hz}), 3.01(\mathrm{dt}, 1 \mathrm{H}, J=5.7,5.4 \mathrm{~Hz}), 4.48(\mathrm{~d}, 1 \mathrm{H}, J=2.0$ $\mathrm{Hz})$, and 7.27-7.38 (m, 5H) ppm; ${ }^{13} \mathrm{C}\left\{{ }^{1} \mathrm{H}\right\} \mathrm{NMR}\left(\mathrm{CDCl}_{3}, 125 \mathrm{MHz}\right): \delta 14.0,22.5,28.1$, 31.3, 57.7, 62.6, 74.4, 126.9, 128.8, 129.4, and $141.2 \mathrm{ppm}$; IR (neat): 3420, 3060, 2957, 2931, 2859, 1713, 1692, 1600, 1578, 1490, 1452, 1408, 1384, and $1314 \mathrm{~cm}^{-1}$; HRMS-ESI $\mathrm{m} / \mathrm{z}$ 189.1275 [(M - $\mathrm{OH})^{+}$; calcd for $\mathrm{C}_{13} \mathrm{H}_{17} \mathrm{O}:$ 189.1279]. erythro-diastereomer: Colorless oil. $[\alpha]_{\mathrm{D}}{ }^{20}=-10.7\left(c=0.43, \mathrm{CHCl}_{3}\right) ;{ }^{1} \mathrm{H} \mathrm{NMR}\left(\mathrm{CDCl}_{3}, 500 \mathrm{MHz}\right): \delta 0.83(\mathrm{t}$, $3 \mathrm{H}, J=7.1 \mathrm{~Hz}), 1.22-1.38(\mathrm{~m}, 4 \mathrm{H}), 1.44-1.56(\mathrm{~m}, 2 \mathrm{H}), 2.22(\mathrm{br} \mathrm{s}, 1 \mathrm{H}), 2.94(\mathrm{t}, 1 \mathrm{H}, J=$ $2.4 \mathrm{~Hz}), 3.13(\mathrm{dt}, 1 \mathrm{H}, J=5.8,5.4 \mathrm{~Hz}), 4.84(\mathrm{~d}, 1 \mathrm{H}, J=2.6 \mathrm{~Hz})$, and $7.28-7.35(\mathrm{~m}, 5 \mathrm{H})$ ppm; ${ }^{13} \mathrm{C}\left\{{ }^{1} \mathrm{H}\right\} \mathrm{NMR}\left(\mathrm{CDCl}_{3}, 125 \mathrm{MHz}\right): \delta 14.0,22.5,28.2,31.3,55.5,61.7,71.4,127.2$, 129.0, 129.4, and $139.6 \mathrm{ppm}$; IR (neat): 3420, 3060, 3025, 2957, 2930, 2859, 1725, $1605,1496,1453,1378,1284,1237$, and $1190 \mathrm{~cm}^{-1}$; HRMS-ESI $\mathrm{m} / z 189.1275[(\mathrm{M}-$ $\mathrm{OH})^{+}$; calcd for $\left.\mathrm{C}_{13} \mathrm{H}_{17} \mathrm{O}: 189.1279\right]$.

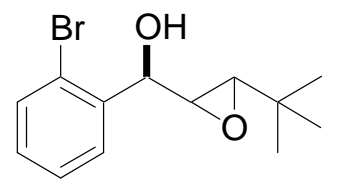

(2-Bromo-phenyl)-(3-tert-butyl-oxiranyl)-methanol (17). The product was prepared by General Procedure B using $50 \mathrm{mg}(0.28$ mmol) $\mathrm{Cy}_{2} \mathrm{BH}, 35 \mu \mathrm{L}$ (0.28 mmol) 3,3-dimethyl-1-butyne, $0.39 \mathrm{~mL}$ $\left(0.78 \mathrm{mmol}, 2.0 \mathrm{M}\right.$ in hexanes) $\mathrm{Et}_{2} \mathrm{Zn}, 2.4 \mathrm{mg}(0.01 \mathrm{mmol})(-)-$ MIB, $29 \mu \mathrm{L}(0.25 \mathrm{mmol}) o$-bromobenzaldehyde, $0.25 \mathrm{~mL}(0.25 \mathrm{mmol}, 1.0 \mathrm{M}$ in hexanes $)$ $\mathrm{Ti}(\mathrm{O} i \mathrm{Pr})_{4}$, and $63 \mu \mathrm{L}(0.30 \mathrm{mmol})(+)$-DIPT. The crude product was purified by column chromatography (5\% ethyl acetate in hexanes) to afford the title compound in $78 \%$ yield $(55 \mathrm{mg}, 0.19 \mathrm{mmol})$. threo-diastereomer: Colorless oil. $[\alpha]_{\mathrm{D}}{ }^{20}=-65.8(c=0.40$, $\left.\mathrm{CHCl}_{3}\right) ;{ }^{1} \mathrm{H} \mathrm{NMR}\left(\mathrm{CDCl}_{3}, 500 \mathrm{MHz}\right): \delta 0.90(\mathrm{~s}, 9 \mathrm{H}), 2.55(\mathrm{~d}, 1 \mathrm{H}, J=5.2 \mathrm{~Hz}), 3.08(\mathrm{~m}$, $2 \mathrm{H}), 5.00(\mathrm{t}, 1 \mathrm{H}, J=4.6 \mathrm{~Hz}), 7.18(\mathrm{t}, 1 \mathrm{H}, J=7.6 \mathrm{~Hz}), 7.37(\mathrm{t}, 1 \mathrm{H}, J=7.5 \mathrm{~Hz}), 7.55(\mathrm{~d}$, $1 \mathrm{H}, J=7.9 \mathrm{~Hz})$, and $7.61(\mathrm{~d}, 1 \mathrm{H}, J=7.3 \mathrm{~Hz}) \mathrm{ppm} ;{ }^{13} \mathrm{C}\left\{{ }^{1} \mathrm{H}\right\} \mathrm{NMR}\left(\mathrm{CDCl}_{3}, 125 \mathrm{MHz}\right): \delta$ 25.7, 30.6, 58.2, 65.2, 71.7, 122.0, 127.88, 127.94, 129.4, 132.8, and $140.0 \mathrm{ppm}$; IR (neat): $3418,3060,2957,2872,2343,1568,1469,1437,1396,1365$, and $1190 \mathrm{~cm}^{-1}$; HRMS-CI $\mathrm{m} / \mathrm{z} 267.0368\left[(\mathrm{M}-\mathrm{OH})^{+}\right.$; calcd for $\mathrm{C}_{13} \mathrm{H}_{16} \mathrm{BrO}$ : 267.0385]. erythrodiastereomer: Colorless oil. $[\alpha]_{\mathrm{D}}{ }^{20}=-55.5\left(c=0.53, \mathrm{CHCl}_{3}\right) ;{ }^{1} \mathrm{H} \mathrm{NMR}\left(\mathrm{CDCl}_{3}, 500\right.$ 
MHz): $\delta 0.84(\mathrm{~s}, 9 \mathrm{H}), 2.42(\mathrm{br} \mathrm{d}, 1 \mathrm{H}, J=2.1 \mathrm{~Hz}), 2.85(\mathrm{br} \mathrm{d}, 1 \mathrm{H}, J=2.3 \mathrm{~Hz}), 3.29(\mathrm{t}$, $1 \mathrm{H}, J=2.4 \mathrm{~Hz}), 5.40($ br s, $1 \mathrm{H}), 7.17(\mathrm{t}, 1 \mathrm{H}, J=7.4 \mathrm{~Hz}), 7.34(\mathrm{t}, 1 \mathrm{H}, J=7.4 \mathrm{~Hz}), 7.51(\mathrm{~d}$, $1 \mathrm{H}, J=7.7 \mathrm{~Hz})$, and $7.54(\mathrm{~d}, 1 \mathrm{H}, J=8.0 \mathrm{~Hz}) \mathrm{ppm} ;{ }^{13} \mathrm{C}\left\{{ }^{1} \mathrm{H}\right\} \mathrm{NMR}\left(\mathrm{CDCl}_{3}, 125 \mathrm{MHz}\right): \delta$ 25.7, 30.3, 56.5, 62.3, 69.1, 122.1, 127.7, 127.9, 129.4, 132.6, and 138.9 ppm; IR (neat): 3426, 3060, 2958, 2861, 1631, 1590, 1586, 1467, 1439, 1390, and $1364 \mathrm{~cm}^{-1}$; HRMS-CI $\mathrm{m} / \mathrm{z} 267.0368\left[(\mathrm{M}-\mathrm{OH})^{+}\right.$; calcd for $\left.\mathrm{C}_{13} \mathrm{H}_{16} \mathrm{BrO}: 267.0385\right]$.

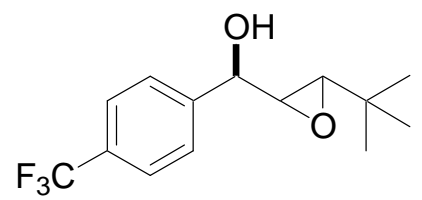

(3-tert-Butyl-oxiranyl)-(4-trifluoromethyl-phenyl)-
methanol (19). The product was prepared by General Procedure B using $50 \mathrm{mg}(0.28 \mathrm{mmol}) \mathrm{Cy}_{2} \mathrm{BH}, 35 \mu \mathrm{L}(0.28$ mmol) 3,3-dimethyl-1-butyne, $0.39 \mathrm{~mL}(0.78 \mathrm{mmol}, 2.0 \mathrm{M}$ in

hexanes $) \quad \mathrm{Et}_{2} \mathrm{Zn}, \quad 2.4 \mathrm{mg} \quad(0.01 \mathrm{mmol}) \quad(-)$-MIB, $34 \quad \mu \mathrm{L} \quad(0.25 \mathrm{mmol}) \quad p$ trifluoromethylbenzaldehyde, $50 \mu \mathrm{L}(0.05 \mathrm{mmol}, 1.0 \mathrm{M}$ in hexanes $) \mathrm{Ti}(\mathrm{O} i \mathrm{Pr})_{4}$, and 10.6 $\mu \mathrm{L}(0.05 \mathrm{mmol})(+)$-DIPT. The crude product was purified by column chromatography ( $5 \%$ ethyl acetate in hexanes) to afford the title compound in $85 \%$ yield $(58 \mathrm{mg}, 0.21$ mmol). threo-diastereomer: Colorless solid. m.p.: $76-79{ }^{\circ} \mathrm{C} ;[\alpha]_{\mathrm{D}}{ }^{20}=-10.0(c=0.27$, $\left.\mathrm{CHCl}_{3}\right) ;{ }^{1} \mathrm{H} \mathrm{NMR}\left(\mathrm{CDCl}_{3}, 500 \mathrm{MHz}\right): \delta 0.90(\mathrm{~s}, 10 \mathrm{H}), 2.89(\mathrm{~d}, 1 \mathrm{H}, J=2.2 \mathrm{~Hz}), 3.03(\mathrm{dd}$, $1 \mathrm{H}, J=5.5,2.2 \mathrm{~Hz}), 4.58(\mathrm{~d}, 1 \mathrm{H}, J=5.4 \mathrm{~Hz}), 7.53(\mathrm{~d}, 2 \mathrm{H}, J=8.1 \mathrm{~Hz})$, and $7.64(\mathrm{~d}, 2 \mathrm{H}, J$ $=8.1 \mathrm{~Hz}) \mathrm{ppm} ;{ }^{13} \mathrm{C}\left\{{ }^{1} \mathrm{H}\right\} \mathrm{NMR}\left(\mathrm{CDCl}_{3}, 125 \mathrm{MHz}\right): \delta 25.7,30.6,59.0,65.3,73.6,125.54$, 125.58, 125.59, and 126.3 ppm; IR (film): 3411, 3028, 2969, 2927, 2871, 2367, 2332, $1622,1477,1460,1420,1365,1331$, and $1241 \mathrm{~cm}^{-1}$; HRMS-CI $\mathrm{m} / z 255.1192\left[(\mathrm{M}-\mathrm{F})^{+}\right.$; calcd for $\mathrm{C}_{14} \mathrm{H}_{17} \mathrm{~F}_{2} \mathrm{O}_{2}$ : 255.1197]. erythro-diastereomer: Colorless solid. m.p.: 74-75 ${ }^{\circ} \mathrm{C} ;[\alpha]_{\mathrm{D}}{ }^{20}=-32.0\left(c=0.10, \mathrm{CHCl}_{3}\right) ;{ }^{1} \mathrm{H} \mathrm{NMR}\left(\mathrm{CDCl}_{3}, 500 \mathrm{MHz}\right): \delta 0.83(\mathrm{~s}, 9 \mathrm{H}), 2.30$ (br s, 1H), $2.93(\mathrm{~d}, 1 \mathrm{H}, J=2.0 \mathrm{~Hz}), 3.03(\mathrm{t}, 1 \mathrm{H}, J=2.6 \mathrm{~Hz}), 4.90(\mathrm{~d}, 1 \mathrm{H}, J=2.2 \mathrm{~Hz})$, $7.48(\mathrm{~d}, 2 \mathrm{H}, J=8.0 \mathrm{~Hz})$, and $7.60(\mathrm{~d}, 2 \mathrm{H}, J=8.0 \mathrm{~Hz}) \mathrm{ppm} ;{ }^{13} \mathrm{C}\left\{{ }^{1} \mathrm{H}\right\} \mathrm{NMR}\left(\mathrm{CDCl}_{3}, 125\right.$ $\mathrm{MHz}): \delta 25.7,31.6,57.9,62.8,75.6,125.50,125.51,125.53$, and $126.6 \mathrm{ppm}$; IR (film): $3350,3037,2955,2868,1661,1620,1477,1463,1414,1365$, and $1327 \mathrm{~cm}^{-1}$; HRMS-CI $\mathrm{m} / \mathrm{z} 255.1192\left[(\mathrm{M}-\mathrm{F})^{+}\right.$; calcd for $\mathrm{C}_{14} \mathrm{H}_{17} \mathrm{~F}_{2} \mathrm{O}_{2}$ : 255.1197].

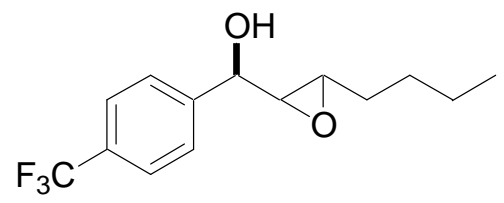

(3-Butyl-oxiranyl)-(4-trifluoromethyl-phenyl)methanol (20). The product was prepared by General Procedure B using $98 \mathrm{mg}(0.55 \mathrm{mmol}) \mathrm{Cy}_{2} \mathrm{BH}, 64 \mu \mathrm{L}$ (0.55 mmol) 1-hexyne, $0.78 \mathrm{~mL}(1.55 \mathrm{mmol}, 2.0 \mathrm{M}$ in hexanes) $\mathrm{Et}_{2} \mathrm{Zn}, 4.8 \mathrm{mg}(0.02 \mathrm{mmol})(-)-\mathrm{MIB}, 68 \mu \mathrm{L}$ $(0.50 \mathrm{mmol}) p$-trifluoromethylbenzaldehyde, $100 \mu \mathrm{L}(0.10 \mathrm{mmol}, 1.0 \mathrm{M}$ in hexanes $)$ $\mathrm{Ti}(\mathrm{OiPr})_{4}$, and $21 \mu \mathrm{L}(0.10 \mathrm{mmol})(+)$-DIPT. The crude product was purified by column chromatography (5\% ethyl acetate in hexanes) to afford the title compound in $76 \%$ yield (104 mg, $0.38 \mathrm{mmol})$. threo-diastereomer: Colorless oil. $[\alpha]_{\mathrm{D}}{ }^{20}=-22.4(c=0.25$, $\left.\mathrm{CHCl}_{3}\right) ;{ }^{1} \mathrm{H} \mathrm{NMR}\left(\mathrm{CDCl}_{3}, 500 \mathrm{MHz}\right): \delta 0.81(\mathrm{t}, 3 \mathrm{H}, J=7.1 \mathrm{~Hz}), 1.19-1.35(\mathrm{~m}, 3 \mathrm{H})$, $1.45-1.53(\mathrm{~m}, 3 \mathrm{H}), 2.37(\mathrm{br} \mathrm{d}, 1 \mathrm{H}, J=3.7 \mathrm{~Hz}), 2.87(\mathrm{dt}, 1 \mathrm{H}, J=5.1,1.8 \mathrm{~Hz}), 3.00(\mathrm{dt}$, $1 \mathrm{H}, J=5.3,1.3 \mathrm{~Hz}), 4.53(\mathrm{br} \mathrm{t}, 1 \mathrm{H}), 7.46(\mathrm{~d}, 2 \mathrm{H}, J=8.0 \mathrm{~Hz})$, and $7.57(\mathrm{~d}, 2 \mathrm{H}, J=8.0$ $\mathrm{Hz}) \mathrm{ppm} ;{ }^{13} \mathrm{C}\left\{{ }^{1} \mathrm{H}\right\} \mathrm{NMR}\left(\mathrm{CDCl}_{3}, 125 \mathrm{MHz}\right): \delta 13.9,22.4,27.9,31.1,57.4,61.8,73.2$, 125.56, 125.59, 126.4, and 144.2 ppm; IR (neat): 3432, 2960, 2931, 2860, 2352, 2326, 1621, 1469, 1456, 1417, 1380, 1326, and $1260 \mathrm{~cm}^{-1}$; HRMS-ESI $\mathrm{m} / z$ 255.1187 [(M - F $)^{+}$; calcd for $\mathrm{C}_{14} \mathrm{H}_{17} \mathrm{~F}_{2} \mathrm{O}_{2}$ : 255.1197]. erythro-diastereomer: Colorless oil. $[\alpha]_{\mathrm{D}}{ }^{20}=-26.0$ 
$\left(c=0.80, \mathrm{CHCl}_{3}\right) ;{ }^{1} \mathrm{H} \mathrm{NMR}\left(\mathrm{CDCl}_{3}, 500 \mathrm{MHz}\right): \delta 0.77(\mathrm{t}, 3 \mathrm{H}, J=7.2 \mathrm{~Hz}), 1.15-1.32(\mathrm{~m}$, $3 \mathrm{H}), 1.38-1.52(\mathrm{~m}, 3 \mathrm{H}), 2.27(\mathrm{br} \mathrm{s}, 1 \mathrm{H}), 2.91(\mathrm{t}, 1 \mathrm{H}, J=2.6 \mathrm{~Hz}), 3.04(\mathrm{dt}, 1 \mathrm{H}, J=5.8,2.0$ $\mathrm{Hz}), 4.87$ (br s, 1H), $7.44(\mathrm{~d}, 2 \mathrm{H}, J=8.0 \mathrm{~Hz})$, and $7.57(\mathrm{~d}, 2 \mathrm{H}, J=8.0 \mathrm{~Hz}) \mathrm{ppm} ;{ }^{13} \mathrm{C}\left\{{ }^{1} \mathrm{H}\right\}$ NMR $\left(\mathrm{CDCl}_{3}, 125 \mathrm{MHz}\right): \delta 13.8,22.3,28.0,31.0,55.2,61.0,70.5,125.49,125.52$, 126.5, and 143.7 ppm; IR (neat): 3425, 3013, 2966, 2932, 2861, 1619, 1457, 1414, 1384, 1326, and $1261 \mathrm{~cm}^{-1}$; HRMS-ESI $\mathrm{m} / z$ 255.1187 $\left[(\mathrm{M}-\mathrm{F})^{+}\right.$; calcd for $\mathrm{C}_{14} \mathrm{H}_{17} \mathrm{~F}_{2} \mathrm{O}_{2}$ : 255.1197].

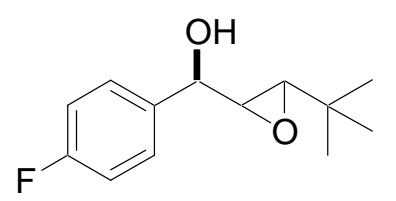

(3-tert-Butyl-oxiranyl)-(4-fluoro-phenyl)-methanol (21). The product was prepared by General Procedure B using $98 \mathrm{mg}(0.55$ mmol) $\mathrm{Cy}_{2} \mathrm{BH}, 68 \mu \mathrm{L}$ (0.55 mmol) 3,3-dimethyl-1-butyne, 0.78 $\mathrm{mL}(1.55 \mathrm{mmol}, 2.0 \mathrm{M}$ in hexanes $) \mathrm{Et}_{2} \mathrm{Zn}, 4.8 \mathrm{mg}(0.02 \mathrm{mmol})$ (-)-MIB, $54 \mu \mathrm{L}(0.50 \mathrm{mmol}) p$-fluorobenzaldehyde, $100 \mu \mathrm{L}(0.10 \mathrm{mmol}, 1.0 \mathrm{M}$ in hexanes) $\mathrm{Ti}(\mathrm{O} i \mathrm{Pr})_{4}$, and $21 \mu \mathrm{L}(0.10 \mathrm{mmol})(+)$-DIPT. The crude product was purified by column chromatography (5\% ethyl acetate in hexanes) to afford the title compound in $92 \%$ yield $(103 \mathrm{mg}, 0.46 \mathrm{mmol})$. threo-diastereomer: Colorless solid. m.p.: $83-85{ }^{\circ} \mathrm{C}$; $[\alpha]_{\mathrm{D}}{ }^{20}=-14.8\left(c=0.23, \mathrm{CHCl}_{3}\right) ;{ }^{1} \mathrm{H} \mathrm{NMR}\left(\mathrm{CDCl}_{3}, 500 \mathrm{MHz}\right): \delta 0.82(\mathrm{~s}, 9 \mathrm{H}), 2.31(\mathrm{br} \mathrm{s}$, $1 \mathrm{H}), 2.78(\mathrm{~d}, 1 \mathrm{H}, J=2.1 \mathrm{~Hz}), 2.95(\mathrm{dd}, 1 \mathrm{H}, J=5.5,2.2 \mathrm{~Hz}), 4.42(\mathrm{~d}, 1 \mathrm{H}, J=5.2 \mathrm{~Hz})$, $6.99(\mathrm{t}, 2 \mathrm{H}, J=8.6 \mathrm{~Hz})$, and $7.31(\mathrm{dd}, 2 \mathrm{H}, J=8.3,5.5 \mathrm{~Hz}) \mathrm{ppm} ;{ }^{13} \mathrm{C}\left\{{ }^{1} \mathrm{H}\right\} \mathrm{NMR}\left(\mathrm{CDCl}_{3}\right.$, $125 \mathrm{MHz}): \delta 25.7,30.6,59.2,65.1,73.6,115.4,115.6,127.68,127.74,136.2$, and 163.5 ppm; IR (neat): 3403, 3002, 2963, 2919, 2872, 1725, 1661, 1637, 1603, 1511, 1461, 1390, 1361, and $1226 \mathrm{~cm}^{-1}$; HRMS-CI $\mathrm{m} / z$ 224.1206 $\left[\mathrm{M}^{+}\right.$; calcd for $\mathrm{C}_{13} \mathrm{H}_{17} \mathrm{FO}_{2}$ : 224.1213]. erythro-diastereomer: Colorless solid. m.p.: $56-59{ }^{\circ} \mathrm{C} ;[\alpha]_{\mathrm{D}}{ }^{20}=-34.3(c=$ $\left.0.40, \mathrm{CHCl}_{3}\right) ;{ }^{1} \mathrm{H} \mathrm{NMR}\left(\mathrm{CDCl}_{3}, 500 \mathrm{MHz}\right): \delta 0.85$ (s, 9H), 2.28 (br s, $\left.1 \mathrm{H}\right), 2.96(\mathrm{~d}, 1 \mathrm{H}, J$ $=2.1 \mathrm{~Hz}), 3.01(\mathrm{t}, 1 \mathrm{H}, J=2.7 \mathrm{~Hz}), 4.84(\mathrm{brd}, 1 \mathrm{H}, J=2.8 \mathrm{~Hz}), 7.04(\mathrm{t}, 2 \mathrm{H}, J=8.5 \mathrm{~Hz})$, and $7.34(\mathrm{dd}, 2 \mathrm{H}, J=8.3,5.5 \mathrm{~Hz}) \mathrm{ppm} ;{ }^{13} \mathrm{C}\left\{{ }^{1} \mathrm{H}\right\} \mathrm{NMR}\left(\mathrm{CDCl}_{3}, 125 \mathrm{MHz}\right): \delta 25.8,30.4$, $58.2,62.7,70.5,115.4,115.6,128.08,128.15,135.6$, and $163.6 \mathrm{ppm}$; IR (KBr): 3409 , $3015,2964,2871,1606,1510,1463,1392,1369,1331$, and $1316 \mathrm{~cm}^{-1}$; HRMS-CI $\mathrm{m} / \mathrm{z}$ 224.1206 [ $\mathrm{M}^{+}$; calcd for $\mathrm{C}_{13} \mathrm{H}_{17} \mathrm{FO}_{2}$ : 224.1213].

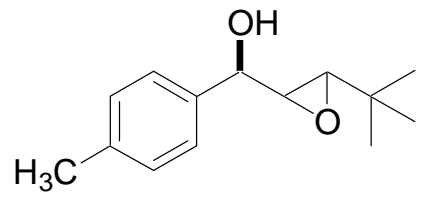

(3-tert-Butyl-oxiranyl)-p-tolyl-methanol (22). The product was prepared by General Procedure B using $98 \mathrm{mg}(0.55$ mmol) $\mathrm{Cy}_{2} \mathrm{BH}, 68 \mu \mathrm{L}(0.55 \mathrm{mmol})$ 3,3-dimethyl-1-butyne, $0.78 \mathrm{~mL}$ (1.55 mmol, 2.0 $\mathrm{M}$ in hexanes) $\mathrm{Et}_{2} \mathrm{Zn}, 4.8 \mathrm{mg}(0.02$ mmol) (-)-MIB, $59 \mu \mathrm{L}(0.50 \mathrm{mmol}) p$-tolualdehyde, $100 \mu \mathrm{L}(0.10 \mathrm{mmol}, 1.0 \mathrm{M}$ in hexanes) $\mathrm{Ti}(\mathrm{O} i \mathrm{Pr})_{4}$, and $21 \mu \mathrm{L}(0.10 \mathrm{mmol})(+)$-DIPT. The crude product was purified by column chromatography (5\% ethyl acetate in hexanes) to afford the title compound in $85 \%$ yield (93 mg, $0.42 \mathrm{mmol}$ ). threo-diastereomer: White solid. m.p.: $36-39{ }^{\circ} \mathrm{C}$; $[\alpha]_{\mathrm{D}}{ }^{20}=-40.0\left(c=0.26, \mathrm{CHCl}_{3}\right) ;{ }^{1} \mathrm{H} \mathrm{NMR}\left(\mathrm{CDCl}_{3}, 500 \mathrm{MHz}\right): \delta 0.81(\mathrm{~s}, 9 \mathrm{H}), 2.28(\mathrm{~s}$, $3 \mathrm{H}), 2.35$ (br d, $1 \mathrm{H}, J=3.9 \mathrm{~Hz}), 2.78(\mathrm{~d}, 1 \mathrm{H}, J=2.2 \mathrm{~Hz}), 2.97(\mathrm{dd}, 1 \mathrm{H}, J=5.5,2.2 \mathrm{~Hz})$, 4.39 (br t, $1 \mathrm{H}), 7.10(\mathrm{~d}, 2 \mathrm{H}, J=7.8 \mathrm{~Hz})$, and $7.22(\mathrm{~d}, 2 \mathrm{H}, J=7.9 \mathrm{~Hz}) \mathrm{ppm} ;{ }^{13} \mathrm{C}\left\{{ }^{1} \mathrm{H}\right\} \mathrm{NMR}$ $\left(\mathrm{CDCl}_{3}, 125 \mathrm{MHz}\right): \delta 21.1,25.7,30.6,59.4,65.1,74.1,125.9,129.2,137.5$, and 137.7 ppm; IR (film): 3416, 3095, 2957, 2867, 1614, 1514, 1482, 1463, 1417, 1393, 1365, 1260, and $1239 \mathrm{~cm}^{-1}$; HRMS-CI $\mathrm{m} / z$ 203.1431 [(M $\left.-\mathrm{OH}\right)^{+}$; calcd for $\mathrm{C}_{14} \mathrm{H}_{19} \mathrm{O}$ : 203.1436]. erythro-diastereomer: White solid. m.p.: $65-68{ }^{\circ} \mathrm{C} ;[\alpha]_{\mathrm{D}}{ }^{20}=-58.2(c=$ 
0.57, $\left.\mathrm{CHCl}_{3}\right) ;{ }^{1} \mathrm{H}$ NMR $\left(\mathrm{CDCl}_{3}, 500 \mathrm{MHz}\right): \delta 0.82(\mathrm{~s}, 9 \mathrm{H}), 2.16(\mathrm{br} \mathrm{s}, 1 \mathrm{H}), 2.28(\mathrm{~s}, 3 \mathrm{H})$, $2.95(\mathrm{~d}, 1 \mathrm{H}, J=2.1 \mathrm{~Hz}), 2.97(\mathrm{t}, 1 \mathrm{H}, J=2.7 \mathrm{~Hz}), 4.76(\mathrm{br} \mathrm{s}, 1 \mathrm{H}), 7.10(\mathrm{~d}, 2 \mathrm{H}, J=7.7$ $\mathrm{Hz})$, and $7.19(\mathrm{~d}, 2 \mathrm{H}, J=8.0 \mathrm{~Hz}) \mathrm{ppm} ;{ }^{13} \mathrm{C}\left\{{ }^{1} \mathrm{H}\right\} \mathrm{NMR}\left(\mathrm{CDCl}_{3}, 125 \mathrm{MHz}\right): \delta 21.2,25.8$, 30.4, 58.3, 62.8, 71.1, 126.5, 129.2, 136.8, and 137.9 ppm; IR (film): 3418, 3018, 2965 , 2923, 2867, 1919, 1515, 1478, 1458, 1412, 1381, and $1322 \mathrm{~cm}^{-1}$; HRMS-CI $\mathrm{m} / \mathrm{z}$ 203.1431 [ $(\mathrm{M}-\mathrm{OH})^{+}$; calcd for $\left.\mathrm{C}_{14} \mathrm{H}_{19} \mathrm{O}: 203.1436\right]$.

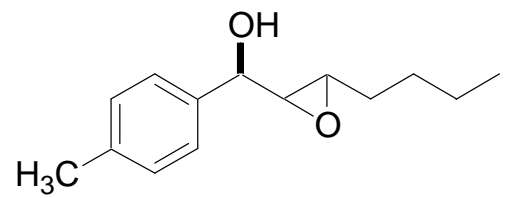

(3-Butyl-oxiranyl)-p-tolyl-methanol (23). The product was prepared by General Procedure B using $89 \mathrm{mg}(0.55$ mmol) $\mathrm{Cy}_{2} \mathrm{BH}, 64 \mu \mathrm{L}(0.55 \mathrm{mmol})$ 1-hexyne, $0.78 \mathrm{~mL}$ $\left(1.55 \mathrm{mmol}, 2.0 \mathrm{M}\right.$ in hexanes) $\mathrm{Et}_{2} \mathrm{Zn}, 4.8 \mathrm{mg}(0.02$ mmol) (-)-MIB, $59 \mu \mathrm{L}(0.50 \mathrm{mmol}) p$-tolualdehyde, 100 $\mu \mathrm{L}(0.10 \mathrm{mmol}, 1.0 \mathrm{M}$ in hexanes $) \mathrm{Ti}(\mathrm{O} i \mathrm{Pr})_{4}$, and $21 \mu \mathrm{L}(0.10 \mathrm{mmol})(+)-\mathrm{DIPT}$. The crude product was purified by column chromatography (5\% ethyl acetate in hexanes) to afford the title compound in $83 \%$ yield $(92 \mathrm{mg}, 0.42 \mathrm{mmol})$. threo-diastereomer: Colorless oil. $[\alpha]_{\mathrm{D}}{ }^{20}=-18.7\left(c=0.31, \mathrm{CHCl}_{3}\right) ;{ }^{1} \mathrm{H} \mathrm{NMR}\left(\mathrm{CDCl}_{3}, 500 \mathrm{MHz}\right): \delta 0.81(\mathrm{t}$, $3 \mathrm{H}, J=7.1 \mathrm{~Hz}), 1.20-1.34(\mathrm{~m}, 4 \mathrm{H}), 1.46-1.51(\mathrm{~m}, 2 \mathrm{H}), 2.25$ (br d, $1 \mathrm{H}, J=4.1 \mathrm{~Hz}), 2.30$ (s, 3H), $2.90(\mathrm{dd}, 1 \mathrm{H}, J=5.2,2.0 \mathrm{~Hz}), 2.97(\mathrm{dt}, 1 \mathrm{H}, J=5.5,1.8 \mathrm{~Hz}), 4.42(\mathrm{br} \mathrm{t}, 1 \mathrm{H}), 7.13$ $(\mathrm{d}, 2 \mathrm{H}, J=7.7 \mathrm{~Hz})$, and $7.23(\mathrm{~d}, 2 \mathrm{H}, J=7.9 \mathrm{~Hz}) \mathrm{ppm} ;{ }^{13} \mathrm{C}\left\{{ }^{1} \mathrm{H}\right\} \mathrm{NMR}\left(\mathrm{CDCl}_{3}, 125 \mathrm{MHz}\right)$ : $\delta 13.9,21.1,22.4,28.0,31.2,57.3,62.2,73.8,126.1,126.4,129.3$, and 137.5 ppm; IR (neat): 3428, 3051, 2961, 2928, 2860, 2356, 1646, 1512, 1462, 1383, 1260, and $1192 \mathrm{~cm}^{-}$ 1; HRMS-CI $m / z$ 202.1362 [(M - $\left.\mathrm{H}_{2} \mathrm{O}\right)^{+}$; calcd for $\mathrm{C}_{14} \mathrm{H}_{18} \mathrm{O}$ : 202.1358]. erythrodiastereomer: Colorless oil. $[\alpha]_{\mathrm{D}}{ }^{20}=-58.4\left(c=0.25, \mathrm{CHCl}_{3}\right) ;{ }^{1} \mathrm{H} \mathrm{NMR}\left(\mathrm{CDCl}_{3}, 500\right.$ $\mathrm{MHz}): \delta 0.79(\mathrm{t}, 3 \mathrm{H}, J=7.1 \mathrm{~Hz}), 1.19-1.33(\mathrm{~m}, 4 \mathrm{H}), 1.43-1.50(\mathrm{~m}, 2 \mathrm{H}), 2.15(\mathrm{br} \mathrm{s}, 1 \mathrm{H})$, $2.28(\mathrm{~s}, 3 \mathrm{H}), 2.89(\mathrm{t}, 1 \mathrm{H}, J=2.5 \mathrm{~Hz}), 3.10(\mathrm{dt}, 1 \mathrm{H}, J=5.6,1.5 \mathrm{~Hz}), 4.77(\mathrm{br} \mathrm{s}, 1 \mathrm{H}), 7.11$ $(\mathrm{d}, 2 \mathrm{H}, J=7.8 \mathrm{~Hz})$, and $7.19(\mathrm{~d}, 2 \mathrm{H}, J=8.1 \mathrm{~Hz}) \mathrm{ppm} ;{ }^{13} \mathrm{C}\left\{{ }^{1} \mathrm{H}\right\} \mathrm{NMR}\left(\mathrm{CDCl}_{3}, 125 \mathrm{MHz}\right)$ : $\delta 13.9,21.2,22.4,28.0,31.2,55.2,61.3,70.9,126.4,129.3,136.7$, and 138.0 ppm; IR (neat): 3430, 3028, 2950, 2916, 2860, 2356, 2334, 1680, 1607, 1512, 1461, 1383, and $1265 \mathrm{~cm}^{-1}$; HRMS-CI $\mathrm{m} / \mathrm{z}, 202.1362$ [ $\left(\mathrm{M}-\mathrm{H}_{2} \mathrm{O}\right)^{+}$; calcd for $\left.\mathrm{C}_{14} \mathrm{H}_{18} \mathrm{O}: 202.1358\right]$.

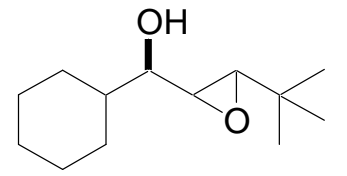

(3-tert-Butyl-oxiranyl)-cyclohexyl-methanol (24). The product was prepared by General Procedure B using $98 \mathrm{mg}(0.55 \mathrm{mmol})$ $\mathrm{Cy}_{2} \mathrm{BH}, 68 \mu \mathrm{L}$ (0.55 mmol) 3,3-dimethyl-1-butyne, $0.78 \mathrm{~mL}$ (1.55 mmol, 2.0 $\mathrm{M}$ in hexanes) $\mathrm{Et}_{2} \mathrm{Zn}, 4.8 \mathrm{mg}$ (0.02 mmol) (-)-MIB, 61 $\mu \mathrm{L}(0.50 \mathrm{mmol})$ cyclohexane carboxaldehyde, $100 \mu \mathrm{L}(0.10 \mathrm{mmol}, 1.0 \mathrm{M}$ in hexanes $)$ $\mathrm{Ti}(\mathrm{O} i \mathrm{Pr})_{4}$, and $21 \mu \mathrm{L}(0.10 \mathrm{mmol})(+)$-DIPT. The crude product was purified by column chromatography (5\% ethyl acetate in hexanes) to afford the title compound in $74 \%$ yield (79 mg, $0.37 \mathrm{mmol})$. threo-diastereomer: White solid. m.p.: $48-50{ }^{\circ} \mathrm{C} ;[\alpha]_{\mathrm{D}}{ }^{20}=+70.7$ $\left(c=0.68, \mathrm{CHCl}_{3}\right) ;{ }^{1} \mathrm{H} \mathrm{NMR}\left(\mathrm{CDCl}_{3}, 500 \mathrm{MHz}\right): \delta 0.92(\mathrm{~s}, 9 \mathrm{H}), 1.00-1.19(\mathrm{~m}, 6 \mathrm{H}), 1.47$ $(\mathrm{m}, 1 \mathrm{H}), 1.64-1.87(\mathrm{~m}, 5 \mathrm{H}), 2.79(\mathrm{~d}, 1 \mathrm{H}, J=2.1 \mathrm{~Hz}), 2.92(\mathrm{t}, 1 \mathrm{H}, J=2.5 \mathrm{~Hz})$, and $3.57(\mathrm{t}$, $1 \mathrm{H}, J=3.1 \mathrm{~Hz}) \mathrm{ppm} ;{ }^{13} \mathrm{C}\left\{{ }^{1} \mathrm{H}\right\} \mathrm{NMR}\left(\mathrm{CDCl}_{3}, 125 \mathrm{MHz}\right): \delta 25.9,26.0,26.2,26.5,28.5$, 28.8, 30.5, 41.8, 56.5, 62.4, and 72.3 ppm; IR (KBr): 3392, 2922, 2853, 1643, 1449, 1391, 1363, 1262, 1245, and $1211 \mathrm{~cm}^{-1}$; HRMS-CI $\mathrm{m} / z 213.1865\left[\mathrm{MH}^{+}\right.$; calcd for $\mathrm{C}_{13} \mathrm{H}_{25} \mathrm{O}_{2}$ : 213.1855]. erythro-diastereomer: White solid. m.p.: $38-48{ }^{\circ} \mathrm{C} ;[\alpha]_{\mathrm{D}}{ }^{20}=$ $-38.9\left(c=0.27, \mathrm{CHCl}_{3}\right) ;{ }^{1} \mathrm{H} \mathrm{NMR}\left(\mathrm{CDCl}_{3}, 500 \mathrm{MHz}\right): \delta 0.92(\mathrm{~s}, 9 \mathrm{H}), 1.03-1.28(\mathrm{~m}, 6 \mathrm{H})$, 
$1.66(\mathrm{~m}, 1 \mathrm{H}), 1.73(\mathrm{~m}, 3 \mathrm{H}), 1.89(\mathrm{~m}, 2 \mathrm{H}), 2.67(\mathrm{~d}, 1 \mathrm{H}, J=2.0 \mathrm{~Hz}), 2.87$ (dd, 1H, $J=5.4$, $2.1 \mathrm{~Hz})$, and $3.13(\mathrm{~m}, 1 \mathrm{H}) \mathrm{ppm} ;{ }^{13} \mathrm{C}\left\{{ }^{1} \mathrm{H}\right\} \mathrm{NMR}\left(\mathrm{CDCl}_{3}, 125 \mathrm{MHz}\right): \delta 25.8,26.0,26.1$, 26.4, 28.6, 28.8, 30.7, 42.2, 57.5, 65.0, and 75.9 ppm; IR (KBr): 3503, 2961, 2929, 2852,

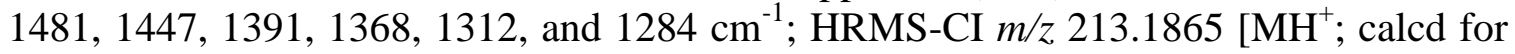
$\left.\mathrm{C}_{13} \mathrm{H}_{25} \mathrm{O}_{2}: 213.1855\right]$.

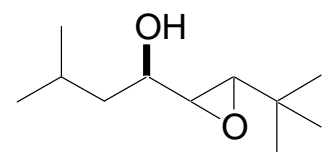

1-(3-tert-Butyl-oxiranyl)-3-methyl-butan-1-ol (25). The product was prepared by General Procedure B using $50 \mathrm{mg}(0.28 \mathrm{mmol})$ $\mathrm{Cy}_{2} \mathrm{BH}, 35 \mu \mathrm{L}$ (0.28 mmol) 3,3-dimethyl-1-butyne, $0.39 \mathrm{~mL}(0.78$ mmol, 2.0 $\mathrm{M}$ in hexanes) $\mathrm{Et}_{2} \mathrm{Zn}, 2.4 \mathrm{mg}$ (0.01 mmol) (-)-MIB, 27 $\mu \mathrm{L}(0.25 \mathrm{mmol})$ isovaleraldehyde, $50 \mu \mathrm{L}(0.05 \mathrm{mmol}, 1.0 \mathrm{M}$ in hexanes $) \mathrm{Ti}(\mathrm{O} i \mathrm{Pr})_{4}$, and $10.6 \mu \mathrm{L}(0.05 \mathrm{mmol})(+)-\mathrm{DIPT}$. The crude product was purified by column chromatography (5\% ethyl acetate in hexanes) to afford the title compound in $89 \%$ yield (42 mg, $0.23 \mathrm{mmol})$. threo-diastereomer: Colorless oil. $[\alpha]_{\mathrm{D}}{ }^{20}=+72.5(c=0.67$, $\left.\mathrm{CHCl}_{3}\right) ;{ }^{1} \mathrm{H} \mathrm{NMR}\left(\mathrm{CDCl}_{3}, 500 \mathrm{MHz}\right): \delta 0.90(\mathrm{~s}, 9 \mathrm{H}), 0.91(\mathrm{~d}, 3 \mathrm{H}, J=6.7 \mathrm{~Hz}), 0.93(\mathrm{~d}$, $3 \mathrm{H}, J=6.7 \mathrm{~Hz}), 1.24-1.29(\mathrm{~m}, 1 \mathrm{H}), 1.36-1.42(\mathrm{~m}, 1 \mathrm{H}), 1.78($ br s, $1 \mathrm{H}), 1.83(\mathrm{~m}, 1 \mathrm{H})$, $2.77(\mathrm{~d}, 1 \mathrm{H}, J=2.3 \mathrm{~Hz}), 2.83(\mathrm{t}, 1 \mathrm{H}, J=2.7 \mathrm{~Hz})$, and $3.86(\mathrm{~m}, 1 \mathrm{H}) \mathrm{ppm} ;{ }^{13} \mathrm{C}\left\{{ }^{1} \mathrm{H}\right\} \mathrm{NMR}$ $\left(\mathrm{CDCl}_{3}, 125 \mathrm{MHz}\right): \delta 22.0,23.5,24.5,25.8,31.6,42.6,58.0,62.3$, and $66.5 \mathrm{ppm}$; IR (neat): $3410,2957,2923,2862,1730,1483,1467,1387,1365,1285,1261$, and $1209 \mathrm{~cm}^{-}$ ${ }^{1}$; HRMS-CI $\mathrm{m} / \mathrm{z} 169.1585\left[(\mathrm{M}-\mathrm{OH})^{+}\right.$; calcd for $\mathrm{C}_{11} \mathrm{H}_{21} \mathrm{O}$ : 169.1592]. erythrodiastereomer: Colorless oil. $[\alpha]_{\mathrm{D}}{ }^{20}=+60.6\left(c=0.53, \mathrm{CHCl}_{3}\right) ;{ }^{1} \mathrm{H} \mathrm{NMR}\left(\mathrm{CDCl}_{3}, 500\right.$ MHz): $\delta 0.90(\mathrm{~s}, 9 \mathrm{H}), 0.93(\mathrm{t}, 6 \mathrm{H}, J=6.8 \mathrm{~Hz}), 1.27-1.31(\mathrm{~m}, 1 \mathrm{H}), 1.49-1.53(\mathrm{~m}, 1 \mathrm{H})$, 1.55 (br s, 1H), 1.79-1.82 (m, 1H), $2.68(\mathrm{~d}, 1 \mathrm{H}, J=2.2 \mathrm{~Hz}), 2.78(\mathrm{dd}, 1 \mathrm{H}, J=5.4,2.3$ $\mathrm{Hz})$, and $3.47(\mathrm{~m}, 1 \mathrm{H}) \mathrm{ppm} ;{ }^{13} \mathrm{C}\left\{{ }^{1} \mathrm{H}\right\} \mathrm{NMR}\left(\mathrm{CDCl}_{3}, 125 \mathrm{MHz}\right): \delta 22.1,23.2,24.3,25.8$, 31.6, 43.2, 59.1, 64.9, and 70.1 ppm; IR (neat): 3379, 2959, 2923, 2872, 1726, 1483, $1463,1417,1361$, and $1255 \mathrm{~cm}^{-1}$; HRMS-CI $\mathrm{m} / z$ 169.1585 [(M $\left.-\mathrm{OH}\right)^{+}$; calcd for $\mathrm{C}_{11} \mathrm{H}_{21} \mathrm{O}:$ 169.1592].

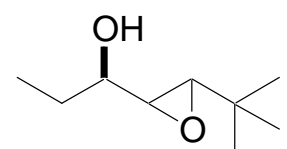

1-(3-tert-Butyl-oxiranyl)-propan-1-ol (26). The product was prepared by General Procedure B using $196 \mathrm{mg}(1.1 \mathrm{mmol}) \mathrm{Cy}_{2} \mathrm{BH}$, $137 \mu \mathrm{L}$ (1.1 mmol) 3,3-dimethyl-1-butyne, $1.1 \mathrm{~mL}(2.1 \mathrm{mmol}, 2.0 \mathrm{M}$ in hexanes) $\mathrm{Et}_{2} \mathrm{Zn}, 9.6 \mathrm{mg}(0.04 \mathrm{mmol})(-)-\mathrm{MIB}, 72 \mu \mathrm{L}(1.0 \mathrm{mmol})$ propanal, $200 \mu \mathrm{L}(0.20 \mathrm{mmol}, 1.0 \mathrm{M}$ in hexanes $) \mathrm{Ti}(\mathrm{O} i \mathrm{Pr})_{4}$, and $42 \mu \mathrm{L}(0.20 \mathrm{mmol})(+)-$ DIPT. The crude product was purified by column chromatography (5\% ethyl acetate in hexanes) to afford the title compound in $69 \%$ yield $(109 \mathrm{mg}, 0.69 \mathrm{mmol})$. threodiastereomer: Colorless oil. $[\alpha]_{\mathrm{D}}{ }^{20}=+80.3\left(c=0.79, \mathrm{CHCl}_{3}\right) ;{ }^{1} \mathrm{H} \mathrm{NMR}\left(\mathrm{CDCl}_{3}, 500\right.$ $\mathrm{MHz}): \delta 0.90(\mathrm{~s}, 9 \mathrm{H}), 0.96(\mathrm{t}, 3 \mathrm{H}, J=7.5 \mathrm{~Hz}), 1.56-1.62(\mathrm{~m}, 2 \mathrm{H}), 1.84(\mathrm{br} \mathrm{d}, 1 \mathrm{H}, J=4.6$ $\mathrm{Hz}), 2.67(\mathrm{~d}, 1 \mathrm{H}, J=2.2 \mathrm{~Hz}), 2.80(\mathrm{dd}, 1 \mathrm{H}, J=5.1,1.8 \mathrm{~Hz})$, and $3.32(\mathrm{~m}, 1 \mathrm{H}) \mathrm{ppm}$; ${ }^{13} \mathrm{C}\left\{{ }^{1} \mathrm{H}\right\} \mathrm{NMR}\left(\mathrm{CDCl}_{3}, 125 \mathrm{MHz}\right): \delta 22.6,25.8,27.4,31.6,58.5,64.7$, and 73.2 ppm; IR (neat): $3401,2955,2851,1728,1602,1578,1462,1370,1367$, and $1273 \mathrm{~cm}^{-1}$; LRMS-CI $\mathrm{m} / \mathrm{z} 141.08\left[(\mathrm{M}-\mathrm{OH})^{+}\right.$; calcd for $\mathrm{C}_{9} \mathrm{H}_{17} \mathrm{O}:$ 141.13]. erythro-diastereomer: Colorless oil. $[\alpha]_{\mathrm{D}}{ }^{20}=+28.0\left(c=0.79, \mathrm{CHCl}_{3}\right) ;{ }^{1} \mathrm{H} \mathrm{NMR}\left(\mathrm{CDCl}_{3}, 500 \mathrm{MHz}\right): \delta 0.90(\mathrm{~s}, 9 \mathrm{H}), 0.99$ $(\mathrm{t}, 3 \mathrm{H}, J=7.5 \mathrm{~Hz}), 1.47-1.60(\mathrm{~m}, 2 \mathrm{H}), 1.79(\mathrm{br} \mathrm{s}, 1 \mathrm{H}), 2.78(\mathrm{~d}, 1 \mathrm{H}, J=2.2 \mathrm{~Hz}), 2.85(\mathrm{t}$, $1 \mathrm{H}, J=2.7 \mathrm{~Hz})$, and $3.72(\mathrm{~m}, 1 \mathrm{H}) \mathrm{ppm} ;{ }^{13} \mathrm{C}\left\{{ }^{1} \mathrm{H}\right\} \mathrm{NMR}\left(\mathrm{CDCl}_{3}, 125 \mathrm{MHz}\right): \delta 22.6,25.8$, 26.6, 31.6, 57.5, 62.4, and 69.6 ppm; IR (neat): 3366, 2955, 2922, 2849, 2367, 2332, 
$1725,1596,1455$, and $1373 \mathrm{~cm}^{-1}$; LRMS-CI $\mathrm{m} / z 141.08\left[(\mathrm{M}-\mathrm{OH})^{+}\right.$; calcd for $\mathrm{C}_{9} \mathrm{H}_{17} \mathrm{O}$ : 141.13].

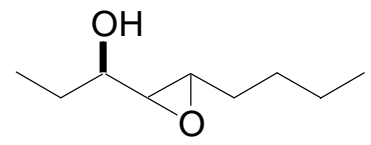

1-(3-Butyl-oxiranyl)-propan-1-ol (27). The product was prepared by General Procedure B using $50 \mathrm{mg}(0.28 \mathrm{mmol})$ $\mathrm{Cy}_{2} \mathrm{BH}, 32 \mu \mathrm{L}(0.28 \mathrm{mmol})$ 1-hexyne, $0.39 \mathrm{~mL}$ (0.78 mmol, 2.0 $\mathrm{M}$ in hexanes) $\mathrm{Et}_{2} \mathrm{Zn}, 2.4 \mathrm{mg}(0.01 \mathrm{mmol})(-)-\mathrm{MIB}, 18 \mu \mathrm{L}(0.25 \mathrm{mmol})$ propanal, $50 \mu \mathrm{L}$ (0.05 mmol, 1.0 M in hexanes) $\mathrm{Ti}(\mathrm{O} i \mathrm{Pr})_{4}$, and $21 \mu \mathrm{L}(0.10 \mathrm{mmol})(+)$-DIPT. The crude product was purified by column chromatography (5\% ethyl acetate in hexanes) to afford the title compound in $68 \%$ yield $(27 \mathrm{mg}, 0.17 \mathrm{mmol})$. threo-diastereomer: Colorless oil. $[\alpha]_{\mathrm{D}}{ }^{20}=-12.5\left(c=0.12, \mathrm{CHCl}_{3}\right) ;{ }^{1} \mathrm{H}$ NMR $\left(\mathrm{CDCl}_{3}, 500 \mathrm{MHz}\right): \delta 0.88(\mathrm{t}, 3 \mathrm{H}, J=7.0$ $\mathrm{Hz}), 0.99$ (t, 3H, $J=7.5 \mathrm{~Hz}), 1.31-1.41(\mathrm{~m}, 4 \mathrm{H}), 1.48-1.62(\mathrm{~m}, 4 \mathrm{H}), 1.76$ (br s, 1H), 2.73 (br t, $1 \mathrm{H}, J=2.7 \mathrm{~Hz}), 2.96(\mathrm{dt}, 1 \mathrm{H}, J=5.7,2.0 \mathrm{~Hz})$, and $3.70(\mathrm{~m}, 1 \mathrm{H}) \mathrm{ppm} ;{ }^{13} \mathrm{C}\left\{{ }^{1} \mathrm{H}\right\}$ NMR $\left(\mathrm{CDCl}_{3}, 125 \mathrm{MHz}\right): \delta 9.6,13.9,22.5,26.5,28.1,31.3,54.9,60.7$, and $69.7 \mathrm{ppm}$; IR (neat): 3356, 2960, 2922, 2852, 2355, 2332, 1729, 1463, 1414, 1378, and $1261 \mathrm{~cm}^{-1}$; HRMS-CI $\mathrm{m} / z$ 141.1273 [(M $-\mathrm{OH})^{+}$; calcd for $\mathrm{C}_{9} \mathrm{H}_{17} \mathrm{O}:$ 141.1279]. erythrodiastereomer: Colorless oil. $[\alpha]_{\mathrm{D}}{ }^{20}=+9.2\left(c=0.41, \mathrm{CHCl}_{3}\right) ;{ }^{1} \mathrm{H} \mathrm{NMR}\left(\mathrm{CDCl}_{3}, 500\right.$ $\mathrm{MHz}): \delta 0.88(\mathrm{t}, 3 \mathrm{H}, J=7.0 \mathrm{~Hz}), 0.97(\mathrm{t}, 3 \mathrm{H}, J=7.5 \mathrm{~Hz}), 1.22-1.27(\mathrm{~m}, 2 \mathrm{H}), 1.30-1.43$ $(\mathrm{m}, 4 \mathrm{H}), 1.48-1.56(\mathrm{~m}, 2 \mathrm{H}), 1.82(\mathrm{br} \mathrm{s}, 1 \mathrm{H}), 2.70(\mathrm{dd}, 1 \mathrm{H}, J=4.9,2.1 \mathrm{~Hz}), 2.87$ (dt, 1H, $J$ $=5.6,2.0 \mathrm{~Hz})$, and $3.35(\mathrm{~m}, 1 \mathrm{H}) \mathrm{ppm} ;{ }^{13} \mathrm{C}\left\{{ }^{1} \mathrm{H}\right\} \mathrm{NMR}\left(\mathrm{CDCl}_{3}, 125 \mathrm{MHz}\right): \delta 9.7,13.9$, 22.5, 27.5, 28.0, 31.3, 56.9, 61.5, and 72.6 ppm; IR (neat): 3424, 2959, 2922, 2851, 2362, 2332, 1731, 1463, 1408, and $1373 \mathrm{~cm}^{-1}$; HRMS-CI $m / z 141.1273\left[(\mathrm{M}-\mathrm{OH})^{+}\right.$; calcd for $\mathrm{C}_{9} \mathrm{H}_{17} \mathrm{O}$ : 141.1279].

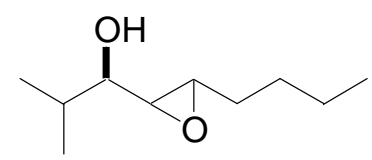

1-(3-Butyl-oxiranyl)-2-methyl-propan-1-ol (28). The product was prepared by General Procedure B using $98 \mathrm{mg}(0.55 \mathrm{mmol})$ $\mathrm{Cy}_{2} \mathrm{BH}, 64 \mu \mathrm{L}$ (0.55 mmol) 1-hexyne, $0.78 \mathrm{~mL}$ (1.55 mmol, 2.0 $\mathrm{M}$ in hexanes) $\mathrm{Et}_{2} \mathrm{Zn}, 4.8 \mathrm{mg}(0.02 \mathrm{mmol})(-)-\mathrm{MIB}, 45 \mu \mathrm{L}(0.50$ mmol) isobutyraldehyde, $100 \mu \mathrm{L}(0.10 \mathrm{mmol}, 1.0 \mathrm{M}$ in hexanes $) \mathrm{Ti}(\mathrm{O} i \mathrm{Pr})_{4}$, and $21 \mu \mathrm{L}$ $(0.10 \mathrm{mmol})(+)$-DIPT. The crude product was purified by column chromatography $(5 \%$ ethyl acetate in hexanes) to afford the title compound in $77 \%$ yield (66 mg, $0.38 \mathrm{mmol})$. threo-diastereomer: Colorless oil. $[\alpha]_{\mathrm{D}}{ }^{20}=-26.3\left(c=0.79, \mathrm{CHCl}_{3}\right) ;{ }^{1} \mathrm{H} \mathrm{NMR}\left(\mathrm{CDCl}_{3}\right.$, $500 \mathrm{MHz}): \delta 0.85(\mathrm{t}, 3 \mathrm{H}, J=7.1 \mathrm{~Hz}), 0.91(\mathrm{~d}, 3 \mathrm{H}, J=6.8 \mathrm{~Hz}), 0.94(\mathrm{~d}, 3 \mathrm{H}, J=6.8 \mathrm{~Hz})$, $1.27-1.41(\mathrm{~m}, 4 \mathrm{H}), 1.47-1.52(\mathrm{~m}, 2 \mathrm{H}), 1.75(\mathrm{~m}, 1 \mathrm{H}), 1.84(\mathrm{~d}, 1 \mathrm{H}, J=5.7 \mathrm{~Hz}), 2.70(\mathrm{dd}$, $1 \mathrm{H}, J=5.0,2.1 \mathrm{~Hz}), 2.82(\mathrm{dt}, 1 \mathrm{H}, J=5.7,1.6 \mathrm{~Hz})$, and $3.10(\mathrm{q}, 1 \mathrm{H}, J=5.7 \mathrm{~Hz}) \mathrm{ppm}$; ${ }^{13} \mathrm{C}\left\{{ }^{1} \mathrm{H}\right\} \mathrm{NMR}\left(\mathrm{CDCl}_{3}, 125 \mathrm{MHz}\right): \delta 13.9,18.1,18.4,22.5,28.0,31.3,32.5,57.1,60.3$, and 76.0 ppm; IR (neat): 3414, 2959, 2930, 2862, 1466, 1381, 1366, 1280, and $1255 \mathrm{~cm}^{-}$ ${ }^{1}$; LRMS-CI $m / z, 155.17$ [(M - OH $)^{+}$; calcd for $\mathrm{C}_{10} \mathrm{H}_{19} \mathrm{O}$ : 155.14]. erythro-diastereomer: Colorless oil. $[\alpha]_{\mathrm{D}}{ }^{20}=+8.1\left(c=0.36, \mathrm{CHCl}_{3}\right) ;{ }^{1} \mathrm{H} \mathrm{NMR}\left(\mathrm{CDCl}_{3}, 500 \mathrm{MHz}\right): \delta 0.85(\mathrm{t}$, $3 \mathrm{H}, J=7.0 \mathrm{~Hz}), 0.93(\mathrm{~d}, 3 \mathrm{H}, J=6.2 \mathrm{~Hz}), 0.94(\mathrm{~d}, 3 \mathrm{H}, J=6.0 \mathrm{~Hz}), 1.29-1.40(\mathrm{~m}, 4 \mathrm{H})$, $1.50(\mathrm{dt}, 2 \mathrm{H}, J=7.2,6.2 \mathrm{~Hz}), 1.75(\mathrm{~m}, 1 \mathrm{H}), 1.78(\mathrm{br} \mathrm{s}, 1 \mathrm{H}), 2.74(\mathrm{t}, 1 \mathrm{H}, J=2.4 \mathrm{~Hz}), 2.94$ $(\mathrm{dt}, 1 \mathrm{H}, J=5.4,2.0 \mathrm{~Hz})$, and $3.51(\mathrm{br} \mathrm{t}, 1 \mathrm{H}) \mathrm{ppm} ;{ }^{13} \mathrm{C}\left\{{ }^{1} \mathrm{H}\right\} \mathrm{NMR}\left(\mathrm{CDCl}_{3}, 125 \mathrm{MHz}\right): \delta$ 13.9, 17.8, 18.5, 22.5, 28.1, 31.3, 31.7, 54.9, 59.6, and 73.0 ppm; IR (neat): 3412, 2963, 2929, 2872, 1640, 1534, 1458, and $1381 \mathrm{~cm}^{-1}$; LRMS-CI $\mathrm{m} / z .155 .17\left[(\mathrm{M}-\mathrm{OH})^{+}\right.$; calcd for $\mathrm{C}_{10} \mathrm{H}_{19} \mathrm{O}$ : 155.14$]$. 
Conditions for the Determination of Enantiomeric Excess. The enantiomeric excess values for the following compounds were determined by chiral HPLC analysis. The conditions for the resolution of the racemates are described below.

$(\boldsymbol{S})$-4,4-Dimethyl-1-phenyl-pent-2-en-1-ol (1): Chiralcel OD-H; $\mathrm{t}_{1}=9.2 \mathrm{~min}, \mathrm{t}_{2}=11.5$ min (hexanes / 2-propanol: 95/5, $0.8 \mathrm{~mL} / \mathrm{min}$ ).

(S)-1-Phenyl-hept-2-en-1-ol (2): Chiralcel OD-H; $\mathrm{t}_{1}=20.3 \mathrm{~min}, \mathrm{t}_{2}=22.5 \mathrm{~min}$ (hexanes / 2-propanol: 98/2, $0.5 \mathrm{~mL} / \mathrm{min}$ ).

(S)-1-(2-Bromo-phenyl)-4,4-dimethyl-pent-2-en-1-ol (3): Chiralcel OD-H; $\mathrm{t}_{1}=13.4$ $\min , \mathrm{t}_{2}=16.4 \mathrm{~min}$ (hexanes / 2-propanol: $97 / 3,0.5 \mathrm{~mL} / \mathrm{min}$ ).

(S)-1-(2-Bromo-phenyl)-hept-2-en-1-ol (4): Chiralcel OD-H; $\mathrm{t}_{1}=15.9 \mathrm{~min}, \mathrm{t}_{2}=19.8$ min (hexanes / 2-propanol: 97/3, $0.5 \mathrm{~mL} / \mathrm{min}$ ).

(S)-1-(4-Trifluoromethyl-phenyl)-hept-2-en-1-ol (6): Chiralcel OB-H; $\mathrm{t}_{1}=23.1 \mathrm{~min}, \mathrm{t}_{2}$ $=31.3 \mathrm{~min}$ (hexanes / 2-propanol: 98/2, $0.5 \mathrm{~mL} / \mathrm{min}$ ).

(S)-4,4-Dimethyl-1-p-tolyl-pent-2-en-1ol (8): Chiralcel OD-H; $\mathrm{t}_{1}=18.8 \mathrm{~min}, \mathrm{t}_{2}=21.8$ min (hexanes / 2-propanol: 98/2, $0.5 \mathrm{~mL} / \mathrm{min}$ ).

(S)-1-p-Tolyl-hept-2-en-1-ol (9): Chiralcel OD-H; $\mathrm{t}_{1}=22.1 \mathrm{~min}, \mathrm{t}_{2}=25.0 \mathrm{~min}$ (hexanes / 2-propanol: $98 / 2,0.5 \mathrm{~mL} / \mathrm{min})$.

(3-tert-Butyl-oxiranyl)-(4-trifluoromethyl-phenyl)-methanol (19): Chiralcel OD-H; $\mathrm{t}_{1}$ $=20.8 \mathrm{~min}, \mathrm{t}_{2}=26.3 \mathrm{~min}$ (hexanes $/ 2$-propanol: $97 / 3,0.5 \mathrm{~mL} / \mathrm{min}$ ).

The enantiomeric excess values for the following compounds were determined by chiral capillary GC analysis using a Supelco $\beta$-Dex 120 fused silica column (30m x 0.25 $\mathrm{mm} \times 0.25 \mu \mathrm{m}$ film thickness). The carrier gas was nitrogen; inlet temperature was 250 ${ }^{\circ} \mathrm{C}$; FID detector at $270{ }^{\circ} \mathrm{C}$. The conditions for the resolution of the racemates are described below.

(S)-1-(4-Fluoro-phenyl)-4,4-dimethyl-pent-2-en-1-ol (7): $t_{1}=371.1 \mathrm{~min}, \mathrm{t}_{2}=376.6$ $\min \left(110^{\circ} \mathrm{C}, 0.5 \mathrm{~mL} / \mathrm{min}\right)$.

(S)-1-Cyclohexyl-4,4-dimethyl-pent-2-en-1-ol (10): $t_{1}=98.4 \mathrm{~min}, \mathrm{t}_{2}=101.5 \mathrm{~min}(110$ ${ }^{\circ} \mathrm{C}, 1.2 \mathrm{~mL} / \mathrm{min}$ ).

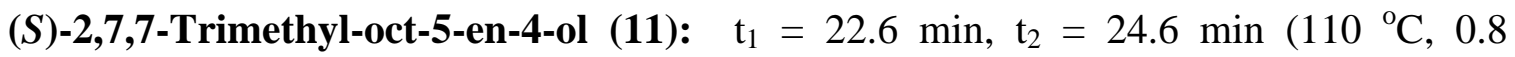
$\mathrm{mL} / \mathrm{min}$ ).

(S)-6,6-Dimethyl-hept-4-en-3-ol (12): $\mathrm{t}_{1}=48.0 \mathrm{~min}, \mathrm{t}_{2}=49.3 \mathrm{~min}\left(80{ }^{\circ} \mathrm{C}, 0.8 \mathrm{~mL} / \mathrm{min}\right)$.

(S)-Non-4-en-3-ol (13): $\mathrm{t}_{1}=79.6 \mathrm{~min}, \mathrm{t}_{2}=81.7 \mathrm{~min}\left(80{ }^{\circ} \mathrm{C}, 0.8 \mathrm{~mL} / \mathrm{min}\right)$. 
(S)-2-Methyl-non-4-en-3-ol (14): $\mathrm{t}_{1}=35.6 \mathrm{~min}, \mathrm{t}_{2}=37.0 \mathrm{~min}\left(100{ }^{\circ} \mathrm{C}, 0.8 \mathrm{~mL} / \mathrm{min}\right)$.

\section{References}

(1) Oppolzer, W.; Radinov, R. N. Helv. Chim. Acta 1992, 75, 170-173. 